\title{
Khovanov module and the detection of unlinks
}

\author{
Matthew HedDeN \\ YI NI
}

\begin{abstract}
We study a module structure on Khovanov homology, which we show is natural under the Ozsváth-Szabó spectral sequence to the Floer homology of the branched double cover. As an application, we show that this module structure detects trivial links. A key ingredient of our proof is that the $\Lambda^{*} H_{1}$-module structure on Heegaard Floer homology detects $S^{1} \times S^{2}$ connected summands.
\end{abstract}

57M27; 57M25

\section{Introduction}

The Jones polynomial [15] has had a tremendous impact since its discovery, leading to an array of invariants of knots and 3-manifolds. The meaning of these invariants is rather elusive. In fact it remains unknown whether there exists a nontrivial knot with the same Jones polynomial as the unknot.

Khovanov discovered a refinement of the Jones polynomial which assigns bigraded homology groups to a link [16]. The Jones polynomial is recovered by taking the Euler characteristic of Khovanov's homology and keeping track of the additional grading by the exponent of a formal variable $q$. One could hope that the geometry contained in this refinement is more transparent, and a step towards understanding the question above would be to determine whether Khovanov homology detects the unknot.

The pure combinatorial nature of Khovanov homology, however, makes direct inspection of its ability to detect the unknot quite difficult. Surprisingly, the most fruitful approach to such questions has been through connections with the world of gauge theory and Floer homology. Indeed, a recent result of Kronheimer and Mrowka uses an invariant of knots arising from instanton Floer homology to prove that Khovanov homology detects the unknot. More precisely, [19, Theorem 1.1] states that

$$
\operatorname{rk} K h^{r}(K)=1 \Longleftrightarrow K \text { is the unknot. }
$$


Their result had numerous antecedents (see Baldwin [1], Baldwin and Levine [2], Baldwin and Plamenevskaya [3], Grigsby and Wehrli [10], the authors [11; 12], the first author and Watson [13] and Roberts [32]), most aimed at generalizing or exploiting a spectral sequence discovered by Ozsváth and Szabó [28] which begins at Khovanov homology and converges to the Heegaard Floer homology of the branched double cover.

Kronheimer and Mrowka's theorem raises the natural question of whether Khovanov homology also detects an unlink of numerous components. Interest in this question is heightened by the existence of infinite families of links which have the same Jones polynomial as unlinks with two or more components; see Eliahou, Kauffman and Thistlethwaite [4] and Thistlethwaite [35]. One immediately observes that the rank of Khovanov homology does not detect unlinks. This is demonstrated by the Hopf link, which has the same rank Khovanov homology as the 2-component unlink (see Section 2.1 for a discussion). Thus more information must be brought to the table if one hopes to use Khovanov homology to detect unlinks. Using a small portion of the bigrading on Khovanov homology we were able to make initial progress on the question of unlink detection in [12]. There we showed [12, Corollary 1.4]

$K h(L)$ detects the unknot $\Longleftrightarrow K h(L)$ detects the two-component unlink,

which, together with Kronheimer and Mrowka's theorem, settles the question for unlinks of two components. Unfortunately, the strategy in [12] could not be extended.

The purpose of this article is to show that Khovanov homology detects unlinks by exploiting a module structure inherent in the theory. In the next section we define a module structure on the Khovanov chain complex, and prove that the induced module structure on the Khovanov homology groups with $\mathbb{F}=\mathbb{Z} / 2 \mathbb{Z}$ coefficients is an invariant of the link. More precisely, we have the following.

Proposition 1 Let $L \subset S^{3}$ be a link of $n$ components. The Khovanov homology $K h(L ; \mathbb{F})$ is a module over the ring

$$
\mathbb{F}\left[X_{0}, \ldots, X_{n-1}\right] /\left(X_{0}^{2}, \ldots, X_{n-1}^{2}\right) .
$$

The isomorphism type of this module is an invariant of $L$.

The action of $X_{i}$ is defined analogously to reduced Khovanov homology [17], and is obtained from the chain map on $C K h(K)$ induced by the link cobordism which merges an unknotted circle to the $i^{\text {th }}$ component of $L$. Our main theorem shows that the Khovanov module detects unlinks. 
Theorem 2 Let $L$ be a link of $n$ components. If there is an isomorphism of modules

$$
K h(L ; \mathbb{F}) \cong \mathbb{F}\left[X_{0}, \ldots, X_{n-1}\right] /\left(X_{0}^{2}, \ldots, X_{n-1}^{2}\right),
$$

then $L$ is the unlink.

Theorem 2 will be proved in the context of the spectral sequence from Khovanov homology to the Heegaard Floer homology of the branched double cover of $L$. This latter invariant also has a module structure. Indeed $\widehat{H F}$ is a module over the exterior algebra on $H^{1}(Y ; \mathbb{F})$, the first singular cohomology of $Y$ with coefficients in $\mathbb{F}$. A key ingredient in our proof, Theorem 4.5, is to refine the Ozsváth-Szabó spectral sequence to incorporate both module structures. A consequence of this result is the following theorem, which indicates the flavor of our refinement.

Theorem 3 There is a spectral sequence of modules, starting at the reduced Khovanov module of the mirror of a link and converging to the Floer homology of its branched double cover.

Armed with this structure, we show that if the Khovanov module is isomorphic to that of the unlink, then the Floer homology of the branched double cover is isomorphic to $\mathbb{F}\left[X_{1}, \ldots, X_{n-1}\right] /\left(X_{1}^{2}, \ldots, X_{n-1}^{2}\right)$ as a module (see Proposition 4.7). The second main ingredient in our proof is the following theorem, which says that Floer homology detects $S^{1} \times S^{2}$ summands in the prime decomposition of a 3-manifold.

Theorem 4 Suppose that $\widehat{H F}(Y ; \mathbb{F}) \cong \mathbb{F}\left[X_{1}, \ldots, X_{n-1}\right] /\left(X_{1}^{2}, \ldots, X_{n-1}^{2}\right)$ as a module. Then $Y \cong M \#\left(\#^{n-1}\left(S^{1} \times S^{2}\right)\right)$, where $M$ is an integer homology sphere satisfying $\widehat{H F}(M) \cong \mathbb{F}$.

This theorem seems interesting in its own right, and complements an array of results on the faithfulness of the Floer invariants for particularly simple manifolds.

The proof of the main theorem follows quickly from Theorems 3 and 4. Indeed, if the Khovanov module of $L$ is isomorphic to that of the unlink, then the two results imply that the branched double cover of $L$ is homeomorphic to the connected sum of copies of $S^{1} \times S^{2}$ with an integer homology sphere whose Floer homology has rank one. Using classical tools from equivariant topology, we then see that $L$ is a split link, each component of which has the Khovanov homology of the unknot. Kronheimer and Mrowka's theorem then tells us that each component is unknotted. 
Outline This paper is organized as follows. In Section 2 we will review Khovanov homology and its module structure. There we prove that the module structure is an invariant. In Section 3 we will give the necessary background on Heegaard Floer homology, especially the module structure and an $A_{\infty}$ type relation. In Section 4 we relate the Heegaard Floer module structure with Ozsváth and Szabó's link surgeries spectral sequence. As an application, we connect the module structure on Khovanov homology of a link with the module structure on the Heegaard Floer homology of the branched double cover of the link. Section 5 is devoted to a nontriviality theorem for the module structure on Heegaard Floer homology. The proof is similar to the standard nontriviality theorem in Heegaard Floer theory. The main theorem is proved in Section 6, using the results in the previous two sections as well as a homological algebra argument.

Acknowledgements This work was initiated when the authors participated the "Homology Theories of Knots and Links" program at MSRI, and was carried out further when the authors visited the Simons Center for Geometry and Physics. We are grateful to Ciprian Manolescu and Tomasz Mrowka for helpful conversations. We also wish to thank Robert Lipshitz, Sucharit Sarkar and the referee for pointing out a mistake in the proof of Proposition 2.2. Special thanks are due to Sucharit Sarkar for suggesting a way to fix the mistake. The first author was partially supported by NSF grant numbers DMS-0906258 and DMS-1150872 and an Alfred P Sloan Research Fellowship. The second author was partially supported by an AIM Five-Year Fellowship, NSF grant numbers DMS-1021956, DMS-1103976 and an Alfred P Sloan Research Fellowship.

\section{Preliminaries on Khovanov homology}

Khovanov homology is a combinatorial invariant of links in the 3-sphere which refines the Jones polynomial. In this section we briefly recall some background on this invariant, but will assume familiarity with [16]. Our primary purpose is to establish notation and define a module structure on the Khovanov chain complex (or homology) which is implicit in [16; 17], but which has not attracted much attention.

To a diagram $D$ of a link $L \subset S^{3}$, Khovanov associates a bigraded cochain complex, $\left(C K h_{i, j}(D), \partial\right)[16]$. The $i$-grading is the cohomological grading, in the sense that is raised by one by the coboundary operator

$$
\text { ว: } C K h_{i, j}(D) \longrightarrow C K h_{i+1, j}(D) .
$$

The $j$-grading is the so-called "quantum" grading, and is preserved by the differential. One can think of this object as a collection of complexes in the traditional sense, with 
the collection indexed by an additional grading. The homology of these complexes does not depend on the particular diagram chosen for $L$, and produces an invariant

$$
K h(L):=\bigoplus_{i, j} K h_{i, j}(L)
$$

called the Khovanov (co)homology of L. Taking the Euler characteristic in each quantum grading, and keeping track of this with a variable $q$, we naturally obtain a Laurent polynomial

$$
V_{K}(q)=\sum_{j}\left(\sum_{i}(-1)^{i} \operatorname{rank} K h_{i, j}(L)\right) \cdot q^{j} .
$$

This polynomial agrees with the (properly normalized) Jones polynomial.

The complex $C K h(D)$ is obtained by applying a $(1+1)$-dimensional TQFT to the hypercube of complete resolutions of the diagram, and the algebra assigned to a single unknotted circle by this structure is $\mathcal{A}=\mathbb{F}[X] /\left(X^{2}\right)$. The product on this algebra is denoted

$$
m: \mathcal{A} \otimes \mathcal{A} \rightarrow \mathcal{A}
$$

There is also a coproduct

$$
\Delta: \mathcal{A} \rightarrow \mathcal{A} \otimes \mathcal{A}
$$

which is defined by letting

$$
\Delta(\mathbf{1})=\mathbf{1} \otimes X+X \otimes \mathbf{1}, \quad \Delta(X)=X \otimes X .
$$

Our purposes will not require strict knowledge of gradings, and for convenience we relax Khovanov homology to a relatively $\mathbb{Z} \oplus \mathbb{Z}$-graded theory. This means that we consider Khovanov homology up to overall shifts in either the homological or quantum grading. In these terms, the quantum grading of $\mathbf{1} \in \mathcal{A}$ is two greater than that of $X$, and the homological grading is given by the number of crossings resolved with a 1 -resolution, in a given complete resolution.

[17, Section 3] describes a module structure on the Khovanov homology of a knot which we now recall. Given a diagram $D$ for a knot $K$, let $p \in K$ be a marked point. Now place an unknotted circle next to $p$ and consider the saddle cobordism that merges the circle with a segment of $D$ neighboring $p$. Cobordisms induce maps between Khovanov complexes and, as such, we have a map

$$
\mathcal{A} \otimes C K h(D) \stackrel{f_{p}}{\longrightarrow} C K h(D) .
$$

Explicitly, the map is the algebra multiplication $\mathcal{A} \otimes \mathcal{A} \rightarrow \mathcal{A}$ between vectors associated to the additional unknot and the unknot in each complete resolution of the diagram 

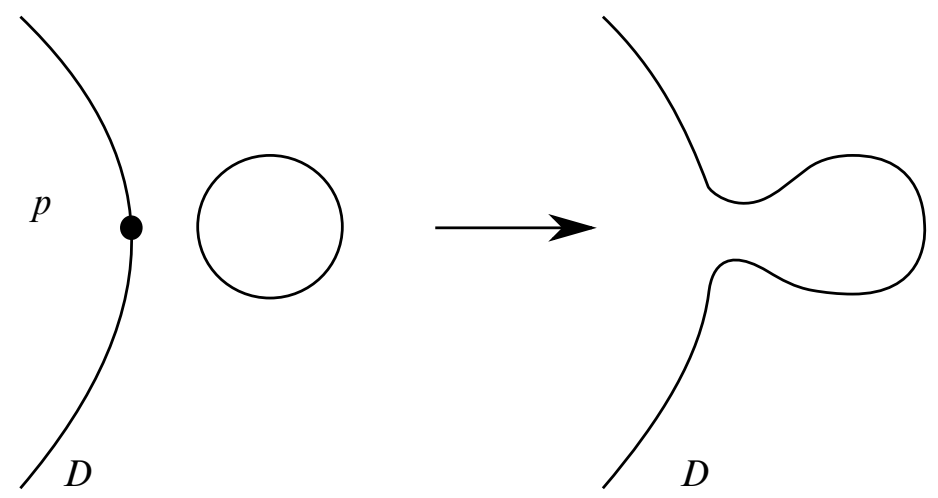

Figure 1: Merging an unknotted circle with $D$

which contains the marked point. We denote the induced map on homology by $F_{p}$. It is independent of the pair $(D, p)$, in a sense made precise by the following proposition.

Proposition 2.1 [17] Let $D$ and $D^{\prime}$ be diagrams for a knot $K$, and let $p \in D$, $p^{\prime} \in D^{\prime}$ be marked points on each diagram. Then there is a commutative diagram

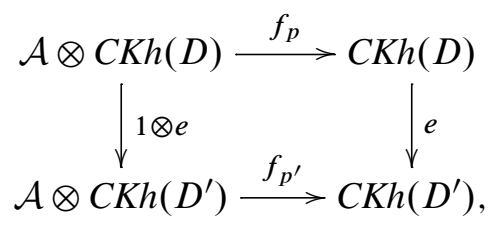

where $e$ is a chain homotopy equivalence.

As observed in [17], the proof is quite simple provided one knows the proof of invariance for Khovanov homology. According to [16], a sequence of Reidemeister moves from $D$ to $D^{\prime}$ induces a chain homotopy equivalence $e$ between the associated complexes. If these moves occur in the complement of a neighborhood of $p$, then $e$ obviously commutes with the map $f_{p}$, where $p$ is regarded as a point in both $D$ and $D^{\prime}$. Now it suffices to observe that Reidemeister moves which cross under or over $p$ can be traded for a sequence of moves which do not (simply drag the segment of knot in question the opposite direction over the 2 -sphere of projection).

Thus a choice of marked point endows the Khovanov homology of a knot with the structure of an $\mathcal{A}$-module. One immediate application of this module structure is that it allows for the definition of the reduced Khovanov homology. Indeed, we can consider $\mathbb{F}=X \cdot \mathcal{A}$ as an $\mathcal{A}$-module, and correspondingly define the reduced Khovanov 
complex ${ }^{1}$

$$
C K h^{r}(D):=C K h(D) \otimes_{\mathcal{A}} \mathbb{F} .
$$

The structure we use is a straightforward generalization of this construction to links. Consider a diagram $D$ for a link $L \subset S^{3}$ of $n$ components. For each component $K_{i} \subset L$ pick a marked point $p_{i} \in K_{i}$ and obtain a map

$$
\mathcal{A} \otimes C K h(D) \stackrel{f_{p_{i}}}{\longrightarrow} C K h(D) .
$$

Now for each $i$, let $x_{i}(-):=f_{p_{i}}(X \otimes-)$ denote the chain map induced from this module structure, and

$$
X_{i}: K h(L) \longrightarrow K h(L), \quad i=0, \ldots, n-1,
$$

denote the map induced on homology. From the definition we see that $x_{i}$, and hence $X_{i}$, commute and satisfy $x_{i}^{2}=0$. Thus we can regard Khovanov homology as a module over $\mathbb{F}\left[X_{0}, \ldots, X_{n-1}\right] /\left(X_{0}^{2}, \ldots, X_{n-1}^{2}\right)$. Showing that this module structure is a link invariant is not as straightforward as above. This is due to the fact that we may not be able to connect two multipointed link diagrams by a sequence of Reidemeister moves which occur in the complement of all the basepoints. One may expect, then, that the module structure is an invariant only of the pointed link isotopy class. In fact the isomorphism type of the module structure on $K h(L ; \mathbb{F})$ does not depend on the choice of marked points or link diagram, as the following proposition shows.

Proposition 2.2 Let $D$ and $D^{\prime}$ be diagrams for a link $L$ of $n$ components and let $p_{i} \in D_{i}, p_{i}^{\prime} \in D_{i}^{\prime}$ be marked points, one chosen on each component of the diagram. Then there is a commutative diagram

$$
\begin{aligned}
& \frac{\mathbb{F}\left[X_{0}, \ldots, X_{n-1}\right]}{\left(X_{0}^{2}, \ldots, X_{n-1}^{2}\right)} \otimes K h(D) \stackrel{f_{p_{*}}}{\longrightarrow} K h(D) \\
& \frac{\mathbb{F}\left[X_{0}, \ldots, X_{n-1}\right]}{\left(X_{0}^{2}, \ldots, X_{n-1}^{2}\right)} \otimes K h\left(D^{\prime}\right) \stackrel{f_{p_{*}^{\prime}}}{\longrightarrow} K h\left(D^{\prime}\right),
\end{aligned}
$$

where $e_{*}$ is an isomorphism induced by a chain homotopy equivalence.

The proof of Proposition 2.2 uses an argument suggested by Sucharit Sarkar.

Any two diagrams $D, D^{\prime}$ are related by a sequence of Reidemeister moves. If a Reidemeister move occurs in the complement of neighborhoods of all marked points $p_{i}$,

\footnotetext{
${ }^{1}$ This is not quite Khovanov's definition, but is isomorphic to it.
} 
then the commutative diagram obviously exists. Thus we only need to consider the case of isotoping an arc over or under a marked point or, equivalently, of sliding a basepoint past a crossing. This case is contained in the following lemma.

Lemma 2.3 Suppose that $D$ is a link diagram, and $\chi_{0}$ is a crossing of $D$. Suppose that $p, q$ are two marked points on a strand passing $\chi_{0}$, such that $p, q$ are separated by $\chi_{0}$. Let $x_{p}, x_{q}: C K h(D) \rightarrow C K h(D)$ be the module multiplications induced by the marked points $p, q$. Then $x_{p}$ and $x_{q}$ are homotopy equivalent.

Consider the cube of resolutions of $D$. If $I J$ is an oriented edge of the cube, let $\partial_{I J}: C(I) \rightarrow C(J)$ be the map induced by the elementary cobordism corresponding to the edge. Let $I_{0}, I_{1}$ be any two complete resolutions of $D$ that differ only at the crossing $\chi_{0}$, where $I_{0}$ is locally the 0 -resolution and $I_{1}$ is locally the 1 -resolution. Suppose that the immediate successors of $I_{0}$ besides $I_{1}$ are $J_{0}^{1}, J_{0}^{2}, \ldots, J_{0}^{r}$. Then the immediate successors of $I_{1}$ are $J_{1}^{1}, J_{1}^{2}, \ldots, J_{1}^{r}$, where $J_{0}^{i}$ and $J_{1}^{i}$ differ only at the crossing $\chi_{0}$.

We write $C K h(D)$ as the direct sum of two subgroups

$$
C K h(D)=C K h\left(D_{0}\right) \oplus C K h\left(D_{1}\right) .
$$

Here $D_{j}$ is the $j$-resolution of $D$ at $\chi_{0}$ for $j=0,1$. Then $C K h\left(D_{1}\right)$ is a subcomplex of $C K h(D)$, and $C K h\left(D_{0}\right)$ is a quotient complex of $C K h(D)$.

We define $H: C K h(D) \rightarrow C K h(D)$ as follows. On $C K h\left(D_{0}\right), H=0$; on $C K h\left(D_{1}\right)$, $H: C K h\left(D_{1}\right) \rightarrow C K h\left(D_{0}\right)$ is defined by the map associated to the elementary cobordism from $D_{1}$ to $D_{0}$. We claim that

$$
x_{p}-x_{q}=\partial H+H \partial .
$$

The claim obviously implies Lemma 2.3, and hence Proposition 2.2. To prove the claim, we begin with another lemma.

Lemma 2.4 Let $I_{0}, I_{1}$ be as above. Then for any $\alpha \in C\left(I_{0}\right)$ and $\beta \in C\left(I_{1}\right)$, the following identities hold:

$$
\begin{aligned}
& \left(x_{p}-x_{q}\right)(\alpha)=H \partial_{I_{0} I_{1}}(\alpha), \\
& \left(x_{p}-x_{q}\right)(\beta)=\partial_{I_{0} I_{1}} H(\beta) .
\end{aligned}
$$

Proof It is easy to check the following identities in $\mathcal{A}$ :

$$
m \Delta=0, \quad \Delta m(a \otimes b)=X a \otimes b+a \otimes X b .
$$

Our conclusion then immediately follows. 
By the definition of $H$, we have $H(\alpha)=0$. Moreover,

$$
H \partial(\alpha)=H\left(\partial_{I_{0} I_{1}} \alpha+\sum_{i=1}^{r} \partial_{I_{0} J_{0}^{i}} \alpha\right)=H \partial_{I_{0} I_{1}}(\alpha),
$$

which is equal to $\left(x_{p}-x_{q}\right)(\alpha)$ by Lemma 2.4. Thus (1) holds for $\alpha \in C\left(I_{0}\right)$.

Turning to $\beta \in C\left(I_{1}\right)$, we have

$$
\begin{gathered}
\partial H(\beta)=\partial_{I_{0} I_{1}} H(\beta)+\sum_{i=1}^{r} \partial_{I_{0} J_{0}^{i}} H(\beta), \\
H \partial(\beta)=H\left(\sum_{i=1}^{r} \partial_{I_{1} J_{1}^{i}}(\beta)\right) .
\end{gathered}
$$

Let $\bar{D}$ be the diagram (of a possibly different link) which is obtained from $D$ by changing the crossing $\chi_{0}$. Then $H$ is the summand of the differential in $C K h(\bar{D})$ which is induced by the edges parallel to $I_{1} I_{0}$. It is clear that

$$
\sum_{i=1}^{r} \partial_{I_{0} J_{0}^{i}} H(\beta)+H\left(\sum_{i=1}^{r} \partial_{I_{1} J_{1}^{i}}(\beta)\right)
$$

is equal to $\partial^{2} \beta$ in $C K h(\bar{D})$, which is zero. So from (2), (3) and Lemma 2.4 we have

$$
(\partial H+H \partial)(\beta)=\partial_{I_{0} I_{1}} H(\beta)=\left(x_{p}-x_{q}\right)(\beta) .
$$

This finishes the proof of (1), hence Lemma 2.3 follows.

Remark Note that we make no claims about the naturality of the module structure. The only result we need is that isotopic links have isomorphic Khovanov modules. Indeed, it seems likely that the module structure on Khovanov homology is functorial, but only in the category of pointed links; that is, links with a choice of basepoint on each component.

When considering the relationship with Heegaard Floer homology, it will be more natural to work in the context of reduced Khovanov homology. Note that reduced Khovanov homology can be interpreted as the homology of the kernel complex $x_{i}: H_{*}(\operatorname{ker}\{C K h(L) \rightarrow C K h(L)\})$ or, equivalently, as the kernel of the map on homology, $\operatorname{ker} X_{i}$; see Shumakovitch [33]. In particular, the module structure from the proposition descends to an $\mathbb{F}\left[X_{1}, \ldots, X_{n-1}\right] /\left(X_{1}^{2}, \ldots, X_{n-1}^{2}\right)$-module structure on the reduced Khovanov homology. Henceforth, we will let $p_{0}$ be the point chosen to define reduced Khovanov homology, so that we have $K h^{r}(L)$ is a module over $\mathbb{F}\left[X_{1}, \ldots, X_{n-1}\right] /\left(X_{1}^{2}, \ldots, X_{n-1}^{2}\right)$. 
Although the reduced Khovanov homology groups do not depend on the choice of component $L_{0}$ containing $p_{0}$, their module structure will, in general, depend on this choice. Thus we use $K h^{r}\left(L, L_{0}\right)$ to emphasize the dependence of the module structure on the component $L_{0}$ and abuse the notation $K^{r}(L)$ when $L_{0}$ is understood.

As we shall see, this module structure on the reduced Khovanov homology is connected to a module structure on the Heegaard Floer homology of the branched double cover through a refinement of the Ozsváth-Szabó spectral sequence.

We also note that, since $X_{i}^{2}=0$, the module structure equips the Khovanov homology (or reduced Khovanov homology) with the structure of a chain complex. Thus it makes sense to talk about the homology of Khovanov homology with respect to $X_{i}$, which we frequently denote $H_{*}\left(K h(L), X_{i}\right)$. It follows from [33] that $H_{*}\left(K h(L), X_{i}\right)=0$ for any $i$. It is far from true, however, that $H_{*}\left(K h^{r}(L), X_{i}\right)=0$ for each $i$, in general.

\subsection{Example: The unlink versus the Hopf link}

It is simple yet instructive to consider the distinction between the Khovanov module of the two component unlink and the Hopf link. The former is represented by Khovanov chain complex $C K h($ Unlink $)=\mathcal{A}\left\langle X_{0}\right\rangle \otimes_{\mathbb{F}} \mathcal{A}\left\langle X_{1}\right\rangle$ with $\partial \equiv 0$. The homology is thus isomorphic to $\mathbb{F}\left[X_{0}, X_{1}\right] /\left(X_{0}^{2}, X_{1}^{2}\right)$ as a module, and is supported in a single homological grading. The reduced Khovanov homology is $K h^{r}$ (Unlink) $\cong \mathbb{F}[X] / X^{2}$.
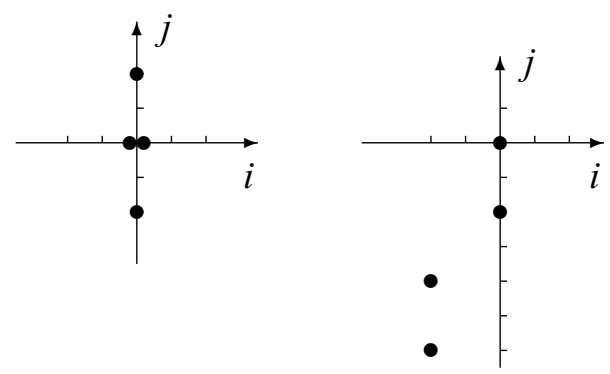

Figure 2: Khovanov homology of the two-component unlink and the Hopf link, where a black dot stands for a copy of $\mathbb{Z}$, the $i$-coordinate is the homological grading and the $j$-coordinate is the quantum grading

The Hopf link, on the other hand, has Khovanov homology

$$
K h(\text { Hopf }) \cong \mathbb{F}\langle a\rangle \oplus \mathbb{F}\langle b\rangle \oplus \mathbb{F}\langle c\rangle \oplus \mathbb{F}\langle d\rangle,
$$

with the relative homological gradings of $c$ and $d$ equal, and two greater than those of $a$ and $b$. The relative quantum grading of $a$ is 2 lower than that of $b$ which is two 


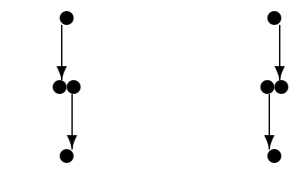

$X_{0}$ action $\quad X_{1}$ action

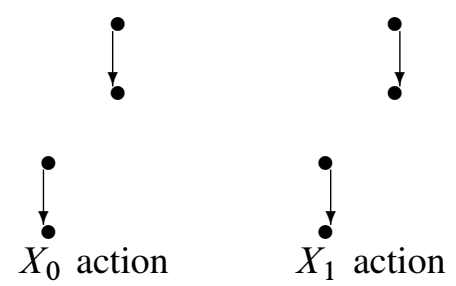

Figure 3: Module structure on the Khovanov homology of the two-component unlink and the Hopf link. The $X_{0}$ and $X_{1}$ actions are different for the twocomponent unlink, but are equal for the Hopf link.

lower than $c$ which, in turn, is two lower than $d$. The module structure is given by

$$
X_{0}(b)=X_{1}(b)=a, \quad X_{0}(d)=X_{1}(d)=c .
$$

More succinctly, the Khovanov module of the Hopf link is isomorphic to

$$
\frac{\mathbb{F}[X]}{X^{2}} \oplus \frac{\mathbb{F}[X]\{2,4\}}{X^{2}},
$$

where we use notation $\{2,4\}$ to denote a shift of 2 and 4 in the homological and quantum gradings respectively. In this module each $X_{i}$ acts as $X$. It follows that the module structure on reduced Khovanov homology is trivial; that is

$$
K h^{r}(\text { Hopf }) \cong \mathbb{F}\langle a\rangle \oplus \mathbb{F}\langle c\rangle,
$$

where $X \in \mathbb{F}[X] / X^{2}$ acts as zero on both summands. Thus, the homology of the reduced Khovanov homology with respect to $X$ is

$$
H_{*}\left(K h^{r}(\text { Hopf }), X\right) \cong \mathbb{F}\langle a\rangle \oplus \mathbb{F}\langle c\rangle,
$$

while for the unlink it is trivial,

$$
H_{*}\left(K h^{r}(\text { Unlink }), X\right)=0 .
$$

\section{Preliminaries on Heegaard Floer homology}

In this section, we recall the basic theory of Heegaard Floer homology, with emphasis on the action of $\Lambda^{*}\left(H_{1}(Y ; \mathbb{Z}) /\right.$ Tors $)$ and twisted coefficients. For a detailed account of the theory, we refer the reader to Ozsváth and Szabó [25] (see also [27; 31; 29] for gentler introductions). 
Suppose that $Y$ is a closed oriented 3-manifold, together with a $\operatorname{Spin}^{c}$ structure $\mathfrak{s} \in \operatorname{Spin}^{c}(Y)$. Let

$$
(\Sigma, \boldsymbol{\alpha}, \boldsymbol{\beta}, z)
$$

be an admissible pointed Heegaard diagram for $(Y, \mathfrak{s})$, in the sense of [25]. To such a diagram we can associate the Ozsváth-Szabó infinity chain complex, $C F^{\infty}(Y, \mathfrak{s})$. This chain complex is freely generated over the ring $\mathbb{F}\left[U, U^{-1}\right]$ by intersection points $\boldsymbol{x} \in \mathbb{T}_{\alpha} \cap \mathbb{T}_{\beta}$, where $\mathbb{T}_{\alpha}$ (resp. $\mathbb{T}_{\beta}$ ) is the Lagrangian torus in $\operatorname{Sym}^{g}(\Sigma)$, the $g$-fold symmetric product of $\Sigma$. The boundary operator is defined by

$$
\partial^{\infty} \boldsymbol{x}=\sum_{\boldsymbol{y} \in \mathbb{T}_{\alpha} \cap \mathbb{T}_{\beta}} \sum_{\left\{\phi \in \pi_{2}(\boldsymbol{x}, \boldsymbol{y}) \mid \mu(\phi)=1\right\}} \# \widehat{\mathcal{M}}(\phi) \cdot U^{n_{z}(\phi)} \boldsymbol{y},
$$

where $\pi_{2}(\boldsymbol{x}, \boldsymbol{y})$ is the set of homotopy classes of Whitney disks connecting $\boldsymbol{x}$ to $\boldsymbol{y}, \mu$ is the Maslov index, $\# \widehat{\mathcal{M}}(\phi)$ denotes the reduction modulo two of the number of unparametrized pseudoholomorphic maps in the homotopy class $\phi$, and $n_{z}(\phi)$ denotes the algebraic intersection number of such a map with the holomorphic hypersurface in $\operatorname{Sym}^{g}(\Sigma)$ consisting of unordered $g$-tuples of points in $\Sigma$ at least one of which is the basepoint $z$.

\subsection{The $\Lambda^{*}\left(H_{1}(Y ; \mathbb{Z}) /\right.$ Tors $)$ action}

In this subsection we describe an action of $\Lambda^{*}\left(H_{1}(Y ; \mathbb{Z}) /\right.$ Tors $)$ on the Floer homology of a 3-manifold $Y$. Let $\zeta \subset \Sigma$ be a closed oriented (possibly disconnected) immersed curve which is in general position with the $\alpha$ - and $\beta$-curves. Namely, $\zeta$ is transverse to these curves, and $\zeta$ does not contain any intersection point of $\alpha$ - and $\beta$-curves. Note that any closed curve $\zeta_{0} \subset Y$ can be homotoped in $Y$ to be an immersed curve in $\Sigma$. If $\phi$ is a topological Whitney disk connecting $\boldsymbol{x}$ to $\boldsymbol{y}$, let $\partial_{\alpha} \phi=(\partial \phi) \cap \mathbb{T}_{\alpha}$. We can also regard $\partial_{\alpha} \phi$ as a multiarc that lies on $\Sigma$ and connects $\boldsymbol{x}$ to $\boldsymbol{y}$. Similarly, we define $\partial_{\beta} \phi$ as a multiarc connecting $\boldsymbol{y}$ to $\boldsymbol{x}$.

Let

$$
a^{\zeta}: C F^{\infty}(Y, \mathfrak{s}) \rightarrow C F^{\infty}(Y, \mathfrak{s})
$$

be defined on generators as

$$
a^{\zeta}(\boldsymbol{x})=\sum_{\boldsymbol{y} \in \mathbb{T}_{\alpha} \cap \mathbb{T}_{\beta}} \sum_{\left\{\phi \in \pi_{2}(\boldsymbol{x}, \boldsymbol{y}) \mid \mu(\phi)=1\right\}}\left(\zeta \cdot\left(\partial_{\alpha} \phi\right)\right) \# \widehat{\mathcal{M}}(\phi) \cdot U^{n_{z}(\phi)} \boldsymbol{y},
$$

where $\zeta \cdot\left(\partial_{\alpha} \phi\right)$ is the algebraic intersection number of $\zeta$ and $\partial_{\alpha} \phi$. We extend the map to the entire complex by requiring linearity and $U$-equivariance. As shown in Ozsváth and Szabó [25, Lemma 4.18], Gromov compactness implies that $a^{\zeta}$ is a chain map. Moreover, this chain map clearly respects the sub, quotient, and subquotient complexes 
$C F^{-}, C F^{+}, \widehat{C F}$, respectively. Thus $a^{\zeta}$ induces a map, denoted $A^{\zeta}=\left(a^{\zeta}\right)_{*}$, on all versions of Heegaard Floer homology.

The next lemma shows $A^{\zeta}$ only depends on the homology class $[\zeta] \in H_{1}(Y ; \mathbb{Z}) /$ Tors.

Lemma 3.1 Suppose $\zeta_{1}, \zeta_{2} \subset \Sigma$ are two curves which are homologous in $H_{1}(Y ; \mathbb{Q})$, then $a^{\zeta_{1}}$ is chain homotopic to $a^{\zeta_{2}}$.

Proof Since $\zeta_{1}$ and $\zeta_{2}$ are homologous in $H_{1}(Y ; \mathbb{Q})$, there exists a nonzero integer $m$ such that $m\left[\zeta_{1}\right]=m\left[\zeta_{2}\right] \in H_{1}(Y ; \mathbb{Z})$. Using the fact that

$$
H_{1}(Y) \cong H_{1}(\Sigma) /\left(\left[\alpha_{1}\right], \ldots,\left[\alpha_{g}\right],\left[\beta_{1}\right], \ldots,\left[\beta_{g}\right]\right),
$$

we conclude that there is a $2-$ chain $B$ in $\Sigma$, such that $\partial B$ consists of $m \zeta_{2}, m\left(-\zeta_{1}\right)$ and copies of $\alpha$-curves and $\beta$-curves. Perturbing $B$ slightly, we get a 2 -chain $B^{\prime}$ such that

$$
\partial B^{\prime}=m \zeta_{2}-m \zeta_{1}+\sum\left(a_{i} \alpha_{i}^{\prime}+b_{i} \beta_{i}^{\prime}\right)
$$

where $\alpha_{i}^{\prime}, \beta_{i}^{\prime}$ are parallel copies of $\alpha_{i}, \beta_{i}$.

Let $\phi$ be a topological Whitney disk connecting $\boldsymbol{x}$ to $\boldsymbol{y}$. Since $\alpha_{i}^{\prime}$ is disjoint from all $\alpha$-curves, we have $\alpha_{i}^{\prime} \cdot \partial_{\alpha} \phi=0$. Similarly,

$$
\beta_{i}^{\prime} \cdot \partial_{\alpha} \phi=-\beta_{i}^{\prime} \cdot \partial_{\beta} \phi=0
$$

We have

$$
n_{\boldsymbol{x}}\left(B^{\prime}\right)-n_{\boldsymbol{y}}\left(B^{\prime}\right)=-\partial B^{\prime} \cdot \partial_{\alpha} \phi=m\left(\zeta_{1}-\zeta_{2}\right) \cdot \partial_{\alpha} \phi \in m \mathbb{Z} .
$$

Pick an intersection point $\boldsymbol{x}_{0}$ representing the $\operatorname{Spin}^{c}$ structure $\mathfrak{s}$. After adding copies of $\Sigma$ to $B^{\prime}$, we can assume that $n_{\boldsymbol{x}_{0}}\left(B^{\prime}\right)$ is divisible by $m$. Since any two intersection points representing $\mathfrak{s}$ are connected by a topological Whitney disk, (4) implies that $n_{\boldsymbol{x}}\left(B^{\prime}\right)$ is divisible by $m$ for any $\boldsymbol{x}$ representing $\mathfrak{s}$.

Now we define a map $H: C F^{\infty}(Y, \mathfrak{s}) \rightarrow C F^{\infty}(Y, \mathfrak{s})$ by letting

$$
H(\boldsymbol{x})=\frac{n_{\boldsymbol{x}}\left(B^{\prime}\right)}{m} \cdot \boldsymbol{x}
$$

on generators, and extending linearly. It follows from (4) that

$$
a^{\zeta_{1}}-a^{\zeta_{2}}=\partial \circ H-H \circ \partial .
$$

Namely, $a^{\zeta_{1}}, a^{\zeta_{2}}$ are chain homotopic. 


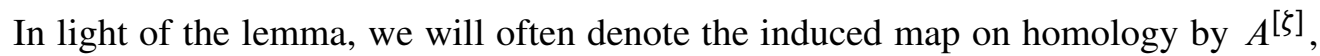
where $[\zeta] \in H_{1}(Y ; \mathbb{Z}) /$ Tors. [25, Lemma 4.17] shows that $A^{[\zeta]}$ satisfies $A^{[\zeta]} \circ A^{[\zeta]}=0$, hence varying the class within $H_{1}(Y ; \mathbb{Z}) /$ Tor endows the Floer homology groups with the structure of a $\Lambda^{*}\left(H_{1}(Y ; \mathbb{Z}) /\right.$ Tors $)$ module. From its definition, the action satisfies $A^{2[\zeta]}=A^{[\zeta]}+A^{[\zeta]}$. In light of the fact that we work with $\mathbb{F}=\mathbb{Z} / 2 \mathbb{Z}$ coefficients, it will thus make more sense to regard the action as by $\Lambda^{*}\left(H_{1}(Y ; \mathbb{F})\right)$, where it is understood that classes in $H_{1}(Y ; \mathbb{F}) \cong H_{1}(Y ; \mathbb{Z}) \otimes \mathbb{F}$ arising from even torsion in $H_{1}(Y ; \mathbb{Z})$ act as zero.

An important example is $Y=\#^{n}\left(S^{1} \times S^{2}\right)$. The module structure of $\widehat{H F}(Y)$ has been computed by Ozsváth and Szabó [24;23]: As a module over $\Lambda^{*}\left(H_{1}(Y ; \mathbb{F})\right)$,

$$
\widehat{H F}(Y) \cong \Lambda^{*}\left(H^{1}(Y ; \mathbb{F})\right),
$$

where the action of $[\zeta]$ is given by the contraction operator $\iota_{[\zeta]}$ defined using the natural hom pairing. We remark that the ring $\Lambda^{*}\left(H_{1}(Y ; \mathbb{F})\right)$ is isomorphic to

$$
\mathbb{F}\left[X_{0}, \ldots, X_{n-1}\right] /\left(X_{0}^{2}, \ldots, X_{n-1}^{2}\right),
$$

and the module $\Lambda^{*}\left(H^{1}(Y ; \mathbb{F})\right)$ is isomorphic to the free module

$$
\mathbb{F}\left[X_{0}, \ldots, X_{n-1}\right] /\left(X_{0}^{2}, \ldots, X_{n-1}^{2}\right) .
$$

As with the module structure on Khovanov homology, we can consider the homology of the Heegaard Floer homology with respect to $A^{[\zeta]}$ :

$$
H_{*}\left(H F^{\circ}(Y, \mathfrak{s}), A^{[\zeta]}\right) \text {. }
$$

For $Y=\#^{n}\left(S^{1} \times S^{2}\right)$, we have $H_{*}\left(\widehat{H F}(Y), X_{i}\right)=0$ for all $i$.

We conclude this subsection by analyzing the $H_{1}(Y ; \mathbb{F})$ action in the presence of essential spheres. We begin with the case of separating spheres. In this case the action splits according to a Künneth principle. More precisely, let

$$
\left(\Sigma_{i}, \boldsymbol{\alpha}_{i}, \boldsymbol{\beta}_{i}, z_{i}\right)
$$

be a Heegaard diagram for $Y_{i}, i=1,2$. Let $\Sigma$ be the connected sum of $\Sigma_{1}$ and $\Sigma_{2}$, with the connected sum performed at $z_{1}$ and $z_{2}$. Let $\zeta_{i} \subset \Sigma_{i} \backslash\left\{z_{i}\right\}$ be a closed curve. Suppose $\mathfrak{s}_{1} \in \operatorname{Spin}^{c}\left(Y_{1}\right), \mathfrak{s}_{2} \in \operatorname{Spin}^{c}\left(Y_{2}\right)$. Now

$$
\left(\Sigma, \boldsymbol{\alpha}_{1} \cup \boldsymbol{\alpha}_{2}, \boldsymbol{\beta}_{1} \cup \boldsymbol{\beta}_{2}, z_{1}=z_{2}\right)
$$

is a Heegaard diagram for $Y_{1} \# Y_{2}$. Using the proof of the Künneth formula for $\widehat{H F}$ of connected sums [24, Proposition 6.1], one sees that the action of $\zeta_{1} \cup \zeta_{2}$ on

$$
\widehat{C F}\left(Y_{1} \# Y_{2}, \mathfrak{s}_{1} \# \mathfrak{s}_{2}\right) \cong \widehat{C F}\left(Y_{1}, \mathfrak{s}_{1}\right) \otimes \widehat{C F}\left(Y_{2}, \mathfrak{s}_{2}\right)
$$


is given by

$$
a^{\zeta_{1} \cup \zeta_{2}}=a^{\zeta_{1}} \otimes \mathrm{id}+\mathrm{id} \otimes a^{\zeta_{2}}
$$

Next, we turn to the case of nonseparating spheres. Here, we have a vanishing theorem for the homology of the Floer homology with respect to the action. First we state a version of the Künneth theorem in this context.

Proposition 3.2 Let $\mathfrak{s}$ be a $\operatorname{Spin}^{c}$ structure on a closed oriented 3-manifold $Y$. Let $\mathfrak{s}_{0}$ be the $\operatorname{Spin}^{c}$ structure on $S^{1} \times S^{2}$ with $c_{1}\left(\mathfrak{s}_{0}\right)=0$. Suppose $\zeta_{1}$ is a closed curve in $Y$, and $\zeta_{2}$ is a closed curve in $S^{1} \times S^{2}$. Then Equation (5) holds on the chain complex

$$
C F^{\circ}\left(Y \#\left(S^{1} \times S^{2}\right), \mathfrak{s} \# \mathfrak{s}_{0}\right) \cong C F^{\circ}(Y, \mathfrak{s}) \otimes \widehat{C F}\left(S^{1} \times S^{2}, \mathfrak{s}_{0}\right) .
$$

Proof This follows from the proof of [24, Proposition 6.4].

We now have the promised vanishing theorem.

Proposition 3.3 Let $\sigma: \mathbb{Z} \rightarrow \mathbb{F}$ be the natural quotient map which sends 1 to $\mathbf{1}$. Let $[\zeta] \in H_{1}(Y ; \mathbb{Z}) /$ Tors be a class for which there exists a two-sphere $S \subset Y$ satisfying $\sigma([\zeta] \cdot[S]) \neq 0 \in \mathbb{F}$. Then

$$
H_{*}\left(\widehat{H F}(Y ; \mathbb{F}), A^{[\zeta]}\right)=0, \quad H_{*}\left(H F^{+}(Y ; \mathbb{F}), A^{[\zeta]}\right)=0 .
$$

Proof Since the sphere $S$ is homologically nontrivial, it is nonseparating. Thus $Y \cong Y_{1} \#\left(S^{1} \times S^{2}\right)$, with $S$ isotopic to $* \times S^{2}$ in $S^{1} \times S^{2}$. We can express [ $\left.\zeta\right]$ as

$$
[\zeta]=\left[\zeta_{1}\right] \oplus\left[\zeta_{2}\right] \in H_{1}\left(Y_{1}\right) \oplus H_{1}\left(S^{1} \times S^{2}\right) \cong H_{1}(Y)
$$

where $\sigma\left(\left[\zeta_{2}\right] \cdot[S]\right) \neq 0$. An explicit calculation with a genus one Heegaard diagram shows that $\widehat{H F}\left(S^{1} \times S^{2}\right)$ has two generators $x, y$ for which

$$
A^{\left[\zeta_{2}\right]}(x)=\left(\left[\zeta_{2}\right] \cdot[S]\right) y, \quad A^{\left[\zeta_{2}\right]}(y)=0 .
$$

Hence $H_{*}\left(\widehat{H F}\left(S^{1} \times S^{2} ; \mathbb{F}\right), A^{\left[\zeta_{2}\right]}\right)=0$. Our conclusion now follows from the previous proposition.

\subsection{Holomorphic polygons and the $\Lambda^{*}\left(H_{1}(Y ; \mathbb{F})\right)$ action}

In this subsection we consider operators on Heegaard Floer homology induced by counting pseudoholomorphic Whitney polygons, and the interaction of these operators with the $H_{1}(Y ; \mathbb{Z}) /$ Tors action. Our main result here is a compatibility relation, Theorem 3.4. This relation will be useful in two ways. First, it will allow us to 
understand the $H_{1}(Y ; \mathbb{Z}) /$ Tors action in the context of the cobordism maps constituting the $(3+1)$-dimensional TQFT in Ozsváth-Szabó theory. Second, it will be the key ingredient for showing that the spectral sequence from Khovanov homology to the Heegaard Floer homology of the branched double cover respects the module structure on both theories.

Let $\left(\Sigma, \eta^{0}, \ldots, \eta^{n}, z\right)$ be a pointed Heegaard $(n+1)$-tuple diagram; that is, a surface of genus $g$, together with $(n+1)$ distinct $g$-tuples $\eta^{i}=\left\{\eta_{1}^{i}, \ldots, \eta_{g}^{i}\right\}$ of homologically linearly independent attaching curves as in [28, Section 4.2]. A Heegaard $(n+1)-$ tuple diagram naturally gives rise to an oriented 4 -manifold $W_{0, \ldots, n}$ by the pants construction [25, Subsection 8.1]. The boundary of this manifold is given by

$$
\partial W_{0, \ldots, n}=-Y_{0,1} \sqcup-Y_{1,2} \sqcup \cdots \sqcup-Y_{n-1, n} \sqcup Y_{0, n},
$$

where $Y_{i, j}$ is the 3 -manifold specified by the attaching curves $\eta^{i}, \eta^{j}$. Each $\eta^{i}$ gives rise to a Lagrangian torus in $\operatorname{Sym}^{g}(\Sigma)$ which we denote $\mathbb{T}_{i}$. We also require an admissibility hypothesis on two-chains with boundary that realize homological relations between curves in the different $g$-tuples $\eta^{i}$, the so-called multiperiodic domains. For this section it suffices to assume that all multiperiodic domains have positive and negative coefficients, a condition which can be achieved by winding their boundary on the Heegaard diagram.

To a Heegaard $(n+1)$-tuple diagram one can associate a chain map

$$
f_{0, \ldots, n}: \bigotimes_{i=1}^{n} \widehat{C F}\left(Y_{i-1, i}\right) \longrightarrow \widehat{C F}\left(Y_{0, n}\right)
$$

defined by counting pseudoholomorphic maps of Whitney $(n+1)$-gons into Sym ${ }^{g}$ with boundary conditions in the Lagrangian tori. On generators, $f_{0, \ldots, n}$ is given by

$$
f_{0, \ldots, n}\left(\boldsymbol{x}_{1} \otimes \cdots \otimes \boldsymbol{x}_{n}\right)=\sum_{\boldsymbol{y}} \sum_{\substack{\phi \in \pi_{2}\left(\boldsymbol{x}_{1}, \ldots, \boldsymbol{x}_{n}, \boldsymbol{y}\right) \\ \mu(\phi)=2-n, n_{z}(\phi)=0}} \# \mathcal{M}(\phi) \cdot \boldsymbol{y},
$$

and we extend linearly to the full complex.

Let us unpack the notation a bit. The first summation is over all $\boldsymbol{y} \in \mathbb{T}_{0} \cap \mathbb{T}_{n}$. In the second, we sum over all homotopy classes of Whitney $(n+1)$-gons. We restrict attention to only those homotopy classes with Maslov index $\mu(\phi)=2-n$. This condition ensures that as we vary within the $(n-2)$-dimensional universal family of conformal $(n+1)$-gons, we obtain a finite number of pseudoholomorphic maps from these conformal polygons to $\operatorname{Sym}^{g}(\Sigma)$. We denote this number, reduced modulo 2 , by $\# \mathcal{M}(\phi)$. When $n=1$ the domain of our pseudoholomorphic map has 
a 1-dimensional automorphism group isomorphic to $\mathbb{R}$, and we consider instead the unparametrized moduli space $\widehat{\mathcal{M}}=\mathcal{M} / \mathbb{R}$; see [25, Section 8] and [28, Section 4] for more details.

The polygon operators satisfy an $A_{\infty}$-associativity relation (see [28, Equation (8)])

$$
\sum_{0 \leq i<j \leq n} f_{0, \ldots, i, j, \ldots, n}\left(\theta_{1} \otimes \cdots \otimes f_{i, \ldots, j}\left(\theta_{i+1} \otimes \cdots \otimes \theta_{j}\right) \otimes \cdots \otimes \theta_{n}\right)=0
$$

where $\theta_{i} \in \widehat{C F}\left(Y_{i-1, i}\right)$ are chains in the Floer complexes associated to the pairs of Lagrangians assigned to the vertices of the $(n+1)$-gon. This relation breaks up into a collection of relations, one for each $n>0$. For $n=1$, the relation simply states that $f_{0,1}: \widehat{C F}\left(Y_{0,1}\right) \rightarrow \widehat{C F}\left(Y_{0,1}\right)$ is a differential.

Examining the definition of the $H_{1}(Y ; \mathbb{Z}) /$ Tor action from the previous section, we see that it closely resembles the Floer differential. Indeed, the action of $\zeta$ is simply the Floer differential weighted by $\zeta \cdot \partial_{0} \phi$, the intersection of a curve representing a class in $H_{1}(Y ; \mathbb{Z}) /$ Tor with the $\eta^{0}$-component of the boundary of the image of a pseudoholomorphic Whitney disk. Motivated by this similarity we define operators for any closed curve $\zeta \in \Sigma$,

$$
a_{0, \ldots, n}^{\zeta}: \bigotimes_{i=1}^{n} \widehat{C F}\left(Y_{i-1, i}\right) \longrightarrow \widehat{C F}\left(Y_{0, n}\right)
$$

On generators, $a_{0, \ldots, n}^{\zeta}$ is given by

$$
a_{0, \ldots, n}^{\zeta}\left(x_{1} \otimes \cdots \otimes x_{n}\right)=\sum_{\boldsymbol{y}} \sum_{\substack{\phi \in \pi_{2}\left(x_{1}, \ldots, x_{n}, y\right) \\ \mu(\phi)=2-n, n_{z}(\phi)=0}}\left(\zeta \cdot\left(\partial_{0} \phi\right)\right) \# \mathcal{M}(\phi) \cdot \boldsymbol{y}
$$

and we extend linearly to the full complex. We have the following $A_{\infty}$-compatibility relation between the $a^{\zeta}$ and $f$ maps.

Theorem 3.4 Let $\zeta \in \Sigma$ be a curve on the Heegaard surface of a given $(n+1)$-tuple diagram which is in general position with respect to all the attaching curves. Let $f_{0, \ldots, n}$ and $a_{0, \ldots, n}^{\zeta}$ be the polygon maps defined above. Then we have the following relation:

$$
\begin{aligned}
\sum_{0 \leq i<j \leq n} a_{0, \ldots, i, j, \ldots, n}^{\zeta}\left(\theta_{1} \otimes \cdots \otimes f_{i, \ldots, j}\left(\theta_{i+1} \otimes \cdots \otimes \theta_{j}\right) \otimes \cdots \otimes \theta_{n}\right) & \\
+ & \sum_{0<j \leq n} f_{0, j, \ldots, n}\left(a_{0, \ldots, j}^{\zeta}\left(\theta_{1} \otimes \cdots \otimes \theta_{j}\right) \otimes \theta_{j+1} \otimes \cdots \otimes \theta_{n}\right)=0
\end{aligned}
$$

where $\theta_{i} \in \widehat{C F}\left(Y_{i-1, i}\right)$ are any Heegaard Floer chains. 
Proof We will consider the $n=3$ version of the relation. A guiding schematic is shown in Figure 4. First we will need to establish some notation. Suppose that $\theta=\sum_{l=1}^{N} \boldsymbol{x}_{l}$ is a chain (recall we work modulo 2, so that no coefficient is needed in front of $\boldsymbol{x}_{i}$ ). Let

$$
\pi_{2}(\theta, \boldsymbol{p}, \boldsymbol{q}, \boldsymbol{s}):=\coprod_{l=1, \ldots, N}\left\{\phi \in \pi_{2}\left(x_{l}, \boldsymbol{p}, \boldsymbol{q}, \boldsymbol{s}\right)\right\},
$$

with similar notation to handle $\pi_{2}(\boldsymbol{p}, \theta, \boldsymbol{q}, \boldsymbol{s}), \pi_{2}(\boldsymbol{s}, \boldsymbol{p}, \theta, \boldsymbol{q})$ and $\pi_{2}(\boldsymbol{p}, \boldsymbol{q}, \boldsymbol{s}, \theta)$.

Consider the moduli space

$$
\mathcal{M}\left(\phi \in \pi_{2}\left(\theta_{1}, \theta_{2}, \theta_{3}, \theta_{4}\right), \mu(\phi)=0\right):=\coprod_{\substack{\phi \in \pi_{2}\left(\theta_{1}, \theta_{2}, \theta_{3}, \theta_{4}\right) \\ \mu(\phi)=0, n_{z}(\phi)=0}} \mathcal{M}(\phi) .
$$

For an appropriate family of almost complex structures on $\operatorname{Sym}^{g}(\Sigma)$, this moduli space is a smooth 1-dimensional manifold with a Gromov compactification. The boundary points of this compactification are in bijection with points in the following products of zero-dimensional moduli spaces:

$$
\begin{aligned}
& \widehat{\mathcal{M}}\left(\phi_{1} \in \pi_{2}\left(\theta_{1}, \rho\right), \mu\left(\phi_{1}\right)=1\right) \times \mathcal{M}\left(\psi_{1} \in \pi_{2}\left(\rho, \theta_{2}, \theta_{3}, \theta_{4}\right), \mu\left(\psi_{1}\right)=-1\right), \\
& \widehat{\mathcal{M}}\left(\phi_{2} \in \pi_{2}\left(\theta_{2}, \rho\right), \mu\left(\phi_{2}\right)=1\right) \times \mathcal{M}\left(\psi_{2} \in \pi_{2}\left(\theta_{1}, \rho, \theta_{3}, \theta_{4}\right), \mu\left(\psi_{2}\right)=-1\right), \\
& \widehat{\mathcal{M}}\left(\phi_{3} \in \pi_{2}\left(\theta_{3}, \rho\right), \mu\left(\phi_{3}\right)=1\right) \times \mathcal{M}\left(\psi_{3} \in \pi_{2}\left(\theta_{1}, \theta_{2}, \rho, \theta_{4}\right), \mu\left(\psi_{3}\right)=-1\right), \\
& \mathcal{M}\left(\phi_{4} \in \pi_{2}\left(\theta_{1}, \theta_{2}, \theta_{3}, \rho\right), \mu\left(\phi_{4}\right)=-1\right) \times \widehat{\mathcal{M}}\left(\psi_{4} \in \pi_{2}\left(\rho, \theta_{4}\right), \mu\left(\psi_{4}\right)=1\right),
\end{aligned}
$$

and

$$
\begin{aligned}
& \mathcal{M}\left(\phi_{5} \in \pi_{2}\left(\theta_{2}, \theta_{3}, \rho\right), \mu\left(\phi_{5}\right)=0\right) \times \mathcal{M}\left(\psi_{5} \in \pi_{2}\left(\theta_{1}, \rho, \theta_{4}\right), \mu\left(\psi_{5}\right)=0\right), \\
& \mathcal{M}\left(\phi_{6} \in \pi_{2}\left(\theta_{1}, \theta_{2}, \rho\right), \mu\left(\phi_{6}\right)=0\right) \times \mathcal{M}\left(\psi_{6} \in \pi_{2}\left(\rho, \theta_{3}, \theta_{4}\right), \mu\left(\psi_{6}\right)=0\right) .
\end{aligned}
$$

In each case $\rho$ is a dummy variable ranging over all chains in the appropriate Floer complex. Therefore we are considering moduli spaces associated to all possible decompositions of the homotopy classes in $\pi_{2}\left(\theta_{1}, \theta_{2}, \theta_{3}, \theta_{4}\right)$ with $\mu(\phi)=0$ and $n_{z}(\phi)=0$ into homotopy classes of bigons concatenated with rectangles and triangles concatenated with triangles. For each $\phi \in \pi_{2}\left(\theta_{1}, \theta_{2}, \theta_{3}, \theta_{4}\right)$ with $\mu(\phi)=0$ we expand the equality

$$
\left(\zeta \cdot \partial_{0} \phi\right) \#(\partial \mathcal{M}(\phi))=0
$$

and observe that since $\phi_{i} * \psi_{i}=\phi$ for each $i=1, \ldots, 6$, we have

$$
\zeta \cdot \partial_{0} \phi=\zeta \cdot \partial_{0} \phi_{i}+\zeta \cdot \partial_{0} \psi_{i}
$$



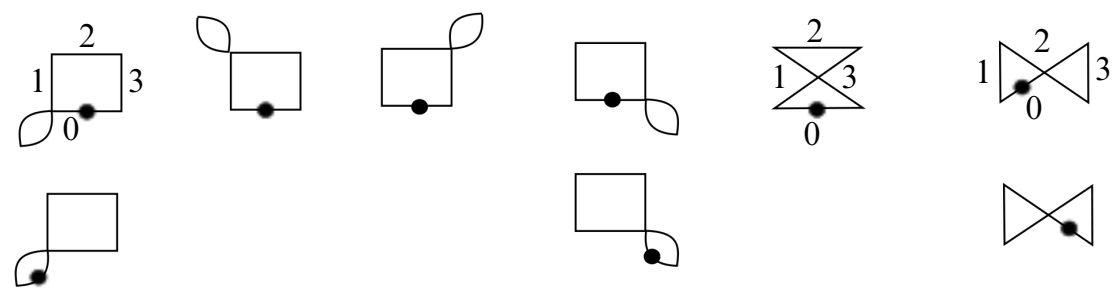

Figure 4: Schematic for $n=3$ case of Theorem 3.4. One-dimensional moduli spaces of rectangles are considered, with boundary conditions in the numbered Lagrangians. The compactified moduli spaces have six types of boundary points. A dark circle on a boundary arc labeled " 0 " indicates that we weight the count of points in a homotopy class by the intersection of the $\eta^{0} \subset \Sigma$ portion of the boundary with the curve $\zeta \subset \Sigma$. Conservation of intersection number ensures that the sum of the weights in any vertical column equals $\zeta \cdot \partial_{0} \phi$. The 9 types of weighted boundary point counts are in bijection with the terms in the $n=3 A_{\infty}$-relation for the $\zeta$-action.

Summing over all $\phi \in \pi_{2}\left(\theta_{1}, \theta_{2}, \theta_{3}, \theta_{4}\right)$, the terms in the expansion correspond to the coefficient of $\theta_{4}$ in

$$
\begin{aligned}
a_{0,1,2,3}^{\zeta}\left(f_{0,1}\left(\theta_{1}\right) \otimes \theta_{2} \otimes\right. & \left.\theta_{3}\right)+f_{0,1,2,3}\left(a_{0,1}^{\zeta}\left(\theta_{1}\right) \otimes \theta_{2} \otimes \theta_{3}\right)+a_{0,1,2,3}^{\zeta}\left(\theta_{1} \otimes f_{1,2}\left(\theta_{2}\right) \otimes \theta_{3}\right) \\
& +a_{0,1,2,3}^{\zeta}\left(\theta_{1} \otimes \theta_{2} \otimes f_{2,3}\left(\theta_{3}\right)\right)+a_{0,3}^{\zeta}\left(f_{0,1,2,3}\left(\theta_{1} \otimes \theta_{2} \otimes \theta_{3}\right)\right) \\
& +f_{0,3}\left(a_{0,1,2,3}^{\zeta}\left(\theta_{1} \otimes \theta_{2} \otimes \theta_{3}\right)\right)+a_{0,1,3}^{\zeta}\left(\theta_{1} \otimes f_{1,2,3}\left(\theta_{2} \otimes \theta_{3}\right)\right) \\
& +a_{0,2,3}^{\zeta}\left(f_{0,1,2}\left(\theta_{1} \otimes \theta_{2}\right) \otimes \theta_{3}\right)+f_{0,2,3}\left(a_{0,1,2}^{\zeta}\left(\theta_{1} \otimes \theta_{2}\right) \otimes \theta_{3}\right) .
\end{aligned}
$$

The terms in the six lines correspond to the contribution from the six types of boundary points for the 1-dimensional moduli space, listed above. Note that the asymmetry between the $a \circ f$ and $f \circ a$ terms is a result of the fact that

$$
\zeta \cdot \partial_{0} \phi_{2}=\zeta \cdot \partial_{0} \phi_{3}=\zeta \cdot \partial_{0} \phi_{5}=0,
$$

which, in turn, is due to the fact that these homotopy classes have boundary conditions outside of $\mathbb{T}_{0}$. This proves the proposition for $n=3$. The general case is a straightforward yet notationally cumbersome extension. 


\subsection{Cobordisms}

In this section we use the compatibility relation between the polygon operators $a^{\zeta}$ and $f$ given by Theorem 3.4 to understand the behavior of the Ozsváth-Szabó cobordism maps with respect to the $H_{1}(Y ; \mathbb{Z})$ / Tors action. Our main goal is Theorem 3.6, which shows that the maps on Floer homology induced by a cobordism $W$ from $Y_{1}$ to $Y_{2}$ commute with the action, provided that the curves in $Y_{1}$ and $Y_{2}$ inducing the action are homologous in $W$.

The key is the $n=2$ compatibility relation. Let $\left(\Sigma, \eta^{0}, \eta^{1}, \eta^{2}, z\right)$ be a pointed Heegaard triple-diagram, and let

$$
f_{0,1,2}: \widehat{C F}\left(Y_{0,1}\right) \otimes \widehat{C F}\left(Y_{1,2}\right) \longrightarrow \widehat{C F}\left(Y_{0,2}\right)
$$

denote the polygon map from the previous section, which counts pseudoholomorphic Whitney triangles. Let $\Theta \in \widehat{C F}\left(Y_{1,2}\right)$ be a cycle. Then we define a map $f_{\Theta}: \widehat{C F}\left(Y_{0,1}\right) \rightarrow \widehat{C F}\left(Y_{0,2}\right)$ by

$$
f_{\Theta}(-):=f_{0,1,2}(-\otimes \Theta) .
$$

The $A_{\infty}$ relation for $n=2$ states that

$$
\begin{aligned}
f_{0,1,2}\left(f_{0,1}(-) \otimes \Theta\right)+f_{0,1,2}\left(-\otimes f_{1,2}(\Theta)\right)+f_{0,2} & \left(f_{0,1,2}(-\otimes \Theta)\right. \\
& =f_{\Theta} \circ \partial_{0,1}+0+\partial_{0,2} \circ f_{\Theta}=0,
\end{aligned}
$$

where we have used the fact that $\Theta$ is a cycle for the $f_{1,2}$ differential. This shows that $f_{\Theta}$ is a chain map. Note that $Y_{1,2}$ could be any 3-manifold for the moment, and $\Theta$ an arbitrary cycle. In many concrete situations $Y_{1,2} \cong \#^{n} S^{1} \times S^{2}$ and $\Theta$ can be assumed to be a generator, $\Theta=y \in \mathbb{T}_{1} \cap \mathbb{T}_{2}$.

Given a closed curve $\zeta \subset \Sigma$ that is in general position with respect to the attaching curves, we can regard $\zeta$ as a curve in $Y_{0,1}$ and $Y_{0,2}$. As such, we have an action by $\zeta$ on $\widehat{C F}\left(Y_{0,1}\right)$ and $\widehat{C F}\left(Y_{0,2}\right)$. The following lemma shows that the induced maps on homology commute with that of $f_{\Theta}$.

Lemma 3.5 Given a pointed Heegaard triple diagram, $\left(\Sigma, \eta^{0}, \eta^{1}, \eta^{2}, z\right)$, we let $f_{\Theta}=f_{0,1,2}(-\otimes \Theta)$ be the chain map from $\widehat{C F}\left(Y_{0,1}\right)$ to $\widehat{C F}\left(Y_{0,2}\right)$ associated to a cycle $\Theta \in \widehat{C F}\left(Y_{1,2}\right)$. Let $\zeta \subset \Sigma$ be a curve and $a^{\zeta_{0}}, a^{\xi_{1}}$ denote the actions of $\zeta$ on $\widehat{C F}\left(Y_{0,1}\right)$ and $\widehat{C F}\left(Y_{0,2}\right)$, respectively. Then $f_{\Theta} \circ a^{\zeta_{0}}$ and $a^{\zeta_{1}} \circ f_{\Theta}$ are chain homotopic. 
Proof The $n=2$ version of Theorem 3.4 states that

$$
\begin{aligned}
a_{0,1,2}^{\zeta}\left(f_{0,1}(-) \otimes \Theta\right)+a_{0,1,2}^{\zeta}(-\otimes & \left.f_{1,2}(\Theta)\right)+a_{0,2}^{\zeta}\left(f_{0,1,2}(-\otimes \Theta)\right) \\
& +f_{0,1,2}\left(a_{0,1}^{\zeta}(-) \otimes \Theta\right)+f_{0,2}\left(a_{0,1,2}^{\zeta}(-) \otimes \Theta\right)=0
\end{aligned}
$$

Let $H(-):=a_{0,1,2}^{\zeta}(-\otimes \Theta)$. Using the fact that $f_{1,2}(\Theta)=0$, we get

$$
H \circ \partial_{0,1}+a^{\zeta_{1}} \circ f_{\Theta}+f_{\Theta} \circ a^{\zeta_{0}}+\partial_{0,2} \circ H=0 .
$$

In other words, $a_{0,1,2}^{\zeta}$ provides the requisite chain homotopy.

The following theorem concerns the naturality of the homological action under the homomorphisms induced by cobordisms. Similar results can be found by Ozsváth and Szabó in [30].

Theorem 3.6 Suppose $Y_{1}, Y_{2}$ are two closed, oriented, connected 3-manifolds, and $W$ is a cobordism from $Y_{1}$ to $Y_{2}$. Let

$$
\widehat{F}_{W}: \widehat{H F}\left(Y_{1}\right) \longrightarrow \widehat{H F}\left(Y_{2}\right)
$$

be the homomorphism induced by $W$. Suppose $\zeta_{1} \subset Y_{1}, \zeta_{2} \subset Y_{2}$ are two closed curves which are homologous in $W$. Then

$$
\widehat{F}_{W} \circ A^{\left[\zeta_{1}\right]}=A^{\left[\zeta_{2}\right]} \circ \widehat{F}_{W}
$$

Proof Since $\zeta_{1}$ and $\zeta_{2}$ are homologous in $W$, there exists an oriented proper surface $S \subset W$ connecting $\zeta_{1}$ to $\zeta_{2}$. By adding tubes between the components of $S$, we can assume that it is connected. Let $S^{\prime}$ denote the surface obtained by removing a collar neighborhood of the components of the boundary of $S$ lying in $Y_{2}$.

Now let $W_{1}^{\prime}$ be a neighborhood of $Y_{1} \cup S^{\prime}$ in $W$. Then $W_{1}^{\prime}$ can be obtained from $Y_{1} \times I$ by adding 1 -handles. Thus $\partial W_{1}^{\prime}=-Y_{1} \sqcup Y_{1}^{\prime}$, where $Y_{1}^{\prime} \cong Y_{1} \#^{k} S^{1} \times S^{2}$. The boundary of $S^{\prime}$ induces a curve $\zeta_{1}^{\prime} \subset Y_{1}^{\prime}$. Using Lemma 3.1 and Proposition 3.2, we see the conclusion of the theorem holds for the cobordism $W_{1}^{\prime}$ and the curves $\zeta_{1}, \zeta_{1}^{\prime}$. The cobordism $W$ can thus be decomposed as $W_{1}^{\prime} \cup_{Y_{1}^{\prime}} W_{2}$, where $W_{2}$ consists of 1-, 2- and 3-handles which are disjoint from $S$; indeed, the portion of $S$ in $W_{2}$ is simply the trace of $\zeta_{1}^{\prime}$ in this cobordism. The map on Floer homology induced by a cobordism is defined by associating chain maps to handle attachments in a handle decomposition [30]. As above, Lemma 3.1 and Proposition 3.2 show that the maps associated to the 1 - and 3-handles in $W_{2}$ commute with the action. Commutativity of the action with the maps associated to the 2-handles is ensured by Lemma 3.5 
(the 2-handle maps are defined as $f_{\Theta}$ for an appropriate Heegaard triple diagram and choice of $\Theta$ ). Hence our conclusion for $W$ holds by the composition law for cobordism maps, [30, Theorem 3.4].

Remark The above theorem has obvious generalizations in two directions. First, one could refine the theorem to account for $\operatorname{Spin}^{c}$ structures: to a cobordism $W$ equipped with a $\operatorname{Spin}^{c}$ structure $\mathfrak{t}$ we have maps between the Floer homology groups $\widehat{H F}\left(Y_{1},\left.\mathfrak{t}\right|_{Y_{1}}\right)$ and $\widehat{H F}\left(Y_{2},\left.\mathfrak{t}\right|_{Y_{2}}\right)$, and one can show that the commutativity theorem respects this structure. Second, one can prove the same theorem for the other versions of Floer homology. In this situation, however, one must take care. We have been considering the sum of cobordism maps associated to all $\operatorname{Spin}^{c}$ structures on $W$. This cannot, in general, be done with the minus and infinity versions of Floer homology, since these versions require admissibility hypotheses that cannot be simultaneously achieved for all $\operatorname{Spin}^{c}$ structures with a single Heegaard diagram. For that reason, the commutativity theorem in these versions must incorporate $\mathrm{Spin}^{c}$ structures. Alternatively, one can continue to sum over all $\operatorname{Spin}^{c}$ structures if we consider the "completed" versions of $C F^{-}$and $C F^{\infty}$ with base rings $\mathbb{F} \llbracket U \rrbracket$ and $\mathbb{F} \llbracket U, U^{-1} \rrbracket$, respectively. Similar remarks hold for the $A_{\infty}$ compatibility relation, Theorem 3.4.

\subsection{Heegaard Floer homology with twisted coefficients}

Suppose $Y$ is a closed oriented $3-$ manifold, $\mathfrak{s} \in \operatorname{Spin}^{c}(Y)$. Let

$$
(\Sigma, \boldsymbol{\alpha}, \boldsymbol{\beta}, z)
$$

be an admissible Heegaard diagram for $(Y, \mathfrak{s})$. Let $\mathcal{R}=\mathbb{F}\left[T, T^{-1}\right]$.

Given $[\omega] \in H^{2}(Y ; \mathbb{Z})$, let $\eta \subset \Sigma$ be a closed curve which represents the Poincaré dual of $\omega$ in $H_{1}(Y ; \mathbb{Z})$. Let $\underline{C F}^{\infty}\left(Y, \mathfrak{s} ; \mathcal{R}_{\eta}\right)$ be the $\mathcal{R}\left[U, U^{-1}\right]$-module freely generated by $\boldsymbol{x} \in \mathbb{T}_{\alpha} \cap \mathbb{T}_{\beta}$ which represent $\mathfrak{s}$. The differential $\underline{\partial}$ is defined by

$$
\underline{\partial}(\boldsymbol{x})=\sum_{\boldsymbol{y}} \sum_{\substack{\phi \in \pi_{2}(\boldsymbol{x}, \boldsymbol{y}) \\ \mu(\phi)=1}} \#(\mathcal{M}(\phi) / \mathbb{R}) T^{\eta \cdot \partial_{\alpha} \phi} U^{n_{z}(\phi)} \boldsymbol{y} .
$$

The homology of this chain complex depends on $\eta$ only through its homology class in $H_{1}(Y)$. Similarly, there are chain complexes $\underline{C F^{ \pm}}\left(Y, \mathfrak{s} ; \mathcal{R}_{\eta}\right)$ and $\widehat{\widehat{C F}}\left(Y, \mathfrak{s} ; \mathcal{R}_{\eta}\right)$. Their homologies are called the $\omega$-twisted Floer homologies, denoted

$$
\underline{H F}^{\circ}\left(Y, \mathfrak{s} ; \mathcal{R}_{\eta}\right) \quad \text { or } \quad \underline{H F}^{\circ}\left(Y, \mathfrak{s} ; \mathcal{R}_{[\omega]}\right)
$$

when there is no confusion. 
The field $\mathbb{F}=\mathcal{R} /(T-1) \mathcal{R}$ is also an $\mathcal{R}$-module. By definition

$$
C F^{\circ}(Y ; \mathbb{F})=\underline{C F^{\circ}}\left(Y ; \mathcal{R}_{[\omega]}\right) \otimes_{\mathcal{R}} \mathbb{F}
$$

is the usual untwisted Heegaard Floer chain complex over $\mathbb{F}$.

There are chain maps on the $\omega$-twisted chain complex induced by cobordisms, as in Ozsváth and Szabó [25; 24], Jabuka and Mark [14] and Ni [21]. More precisely, suppose $W: Y_{1} \rightarrow Y_{2}$ is a cobordism, $[\Omega] \in H^{2}(W ; \mathbb{Z})$. Let $\left[\omega_{1}\right],\left[\omega_{2}\right]$ be the restriction of $[\Omega]$ to $Y_{1}$ and $Y_{2}$, respectively. Then there is a map

$$
\underline{F}_{W ;[\Omega]}^{\circ}: \underline{H F}^{\circ}\left(Y_{1} ; \mathcal{R}_{\left[\omega_{1}\right]}\right) \longrightarrow \underline{H F}^{\circ}\left(Y_{2} ; \mathcal{R}_{\left[\omega_{2}\right]}\right) .
$$

We can also define the $\Lambda^{*}\left(H_{1}(Y) /\right.$ Tors $)$ action on the $\omega$-twisted Floer homology by letting

$$
a^{\zeta}(\boldsymbol{x})=\sum_{\boldsymbol{y} \in \mathbb{T}_{\alpha} \cap \mathbb{T}_{\beta}} \sum_{\left\{\phi \in \pi_{2}(\boldsymbol{x}, \boldsymbol{y}) \mid \mu(\phi)=1\right\}}\left(\zeta \cdot\left(\partial_{\alpha} \phi\right)\right) \# \widehat{\mathcal{M}}(\phi) T^{\eta \cdot \partial_{\alpha} \phi} U^{n_{z}(\phi)} \boldsymbol{y} .
$$

As in Section 3.3, there are twisted versions of (5) and Theorem 3.6.

\section{Module structures and the link surgeries spectral sequence}

The connection between Khovanov homology and Heegaard Floer homology arises from a calculational tool called the link surgeries spectral sequence, [28, Theorem 4.1]. Roughly, this device takes as input a framed link in a 3-manifold, with output a filtered complex. The homology of the complex is isomorphic to the Heegaard Floer homology of the underlying 3-manifold, and the $E_{1}$ term of the associated spectral sequence splits as a direct sum of the Heegaard Floer groups of the manifolds obtained by surgery on the link with varying surgery slopes. The differentials in the spectral sequence are induced by the holomorphic polygon maps of Section 3.2. Applying this machinery to a particular surgery presentation of the 2 -fold branched cover of a link produces the spectral sequence from Khovanov homology to the Heegaard Floer homology.

Our purpose in this section is to refine the link surgeries spectral sequence to incorporate the $\Lambda^{*}\left(H_{1}(Y ; \mathbb{Z}) /\right.$ Tors $)$-module structure on Floer homology, and subsequently relate this structure to the module structure on Khovanov homology. In Section 4.2 we prove that a curve in the complement of a framed link induces a filtered chain map acting on the complex giving rise to the link surgeries spectral sequence, Theorem 4.3. In Section 4.3 we use these filtered chain maps to endow the spectral sequence from Khovanov homology to the Floer homology of the branched double cover with a module structure. This module structure allows us to prove a "collapse" result for the 
spectral sequence (Proposition 4.7). This result states that if the Khovanov module of a link is isomorphic to that of the unlink then this module is also isomorphic to the Floer homology of the branched double cover. Since our results involve filtered chain maps and the morphisms they induce on spectral sequences, we begin with a digression on spectral sequences and their morphisms. The reader familiar with these concepts may wish to skip ahead to Section 4.2, but is warned that our perspective on spectral sequences, while equivalent to the standard treatment, is slightly nonstandard.

\subsection{A review of spectral sequences and their morphisms}

Suppose that a complex of vector spaces $(C, d)$ admits a decomposition

$$
C=\bigoplus_{i \geq 0} C^{(i)}
$$

which is respected by the differential, in the sense that

$$
d=d^{(0)}+d^{(1)}+d^{(2)}+\cdots,
$$

where $d^{(m)}: C^{(i)} \rightarrow C^{(i+m)}$ for each $i$. From this structure, one can construct a spectral sequence; that is, a sequence of chain complexes $\left\{\left(E_{r}, \delta_{r}\right)\right\}_{r=0}^{\infty}$ satisfying $H_{*}\left(E_{r}, \delta_{r}\right) \cong E_{r+1}$. Under mild assumptions, one has $\delta_{r}=0$ for all sufficiently large $r$, and the resulting limit satisfies $E_{\infty} \cong H_{*}(C, d)$. Typically, one constructs such a spectral sequence by noting that the subcomplexes,

$$
F^{p}=\bigoplus_{i \geq p} C^{(i)}
$$

form a filtration $C=F^{0} \supset F^{1} \supset \ldots$, and then appealing to the well-known construction of the spectral sequence associated to a filtered complex (see, eg McCleary [20]).

For the purpose of understanding morphisms of spectral sequence induced by filtered chain maps, we find it more transparent to construct the spectral sequence by a method called iterative cancellation or reduction, a procedure we briefly recall. The method relies on the following well-known lemma.

Lemma 4.1 Let $(C, d)$ be a chain complex of $R$-modules, freely generated by chains $\left\{\boldsymbol{x}_{i}\right\}_{i \in I}$, and suppose that $d\left(\boldsymbol{x}_{k}, \boldsymbol{x}_{l}\right)=1$, where $d(\boldsymbol{a}, \boldsymbol{b})$ denotes the coefficient of $\boldsymbol{b}$ in $d(\boldsymbol{a})$. Then we can define a complex $\left(C^{\prime}, d^{\prime}\right)$, freely generated by $\left\{\boldsymbol{x}_{i} \mid i \neq k, l\right\}$, which is chain homotopy equivalent to $(C, d)$. 
Proof Let $h: C \rightarrow C$ be the module homomorphism defined by $h\left(x_{l}\right)=x_{k}$ and $h\left(\boldsymbol{x}_{i}\right)=0$ if $i \neq l$. Then the differential on $C^{\prime}$ is given by

$$
d^{\prime}=\pi \circ(d-d h d) \circ \iota,
$$

where $\pi: C \rightarrow C^{\prime}$ and $\iota: C^{\prime} \rightarrow C$ are the natural projection and inclusions. Now the chain maps $f: C \rightarrow C^{\prime}$ and $g: C^{\prime} \rightarrow C$ defined by

$$
f=\pi \circ(\mathbb{I}-d \circ h) g=(\mathbb{I}-h \circ d) \circ \iota,
$$

are mutually inverse chain homotopy equivalences. Indeed, $f \circ g=\mathbb{I}_{C^{\prime}}$ and $g \circ f \sim \mathbb{I}_{C}$ via the homotopy $h$.

Of course we can employ the lemma under the weaker assumption that $d\left(\boldsymbol{x}_{k}, \boldsymbol{x}_{l}\right)$ is a unit in $R$, simply by rescaling the basis. In the present situation $(C, d)$ is a complex of vector spaces, so this applies whenever $d\left(\boldsymbol{x}_{k}, \boldsymbol{x}_{l}\right) \neq 0$.

We will use the lemma in a filtered sense. To make this precise, given a complex with increasing filtration as above, let $\mathcal{F}(a) \in \mathbb{Z}^{\geq 0}$ denote the filtration level of a chain, ie

$$
\mathcal{F}(a)=\max \left\{i \in \mathbb{Z}^{\geq 0} \mid a \in F^{i}\right\},
$$

and define $\mathcal{F}(a, b)=\mathcal{F}(a)-\mathcal{F}(b)$. Note that the filtration of a linear combination of chains satisfies

$$
\mathcal{F}(a+b) \geq \min \{\mathcal{F}(a), \mathcal{F}(b)\}
$$

Lemma 4.2 With the notation from Lemma 4.1, suppose that $(C, d)$ is a filtered complex, that $d\left(\boldsymbol{x}_{k}, \boldsymbol{x}_{l}\right)=1$ and that

$$
\mathcal{F}\left(d\left(x_{k}\right), x_{l}\right) \geq 0 .
$$

Then the reduced complex $\left(C^{\prime}, d^{\prime}\right)$ inherits a filtration $\mathcal{F}^{\prime}$ from $(C, d)$ by the formula $\mathcal{F}^{\prime}(a):=\mathcal{F}(\iota(a))$ and the filtration degree of $d^{\prime}$ is no less than that of $d$ in the sense that

$$
\mathcal{F}^{\prime}\left(d^{\prime}(a)\right) \geq \mathcal{F}(d \circ \iota(a)) \text { for all } a \in C^{\prime} .
$$

Moreover, if $\mathcal{F}\left(\boldsymbol{x}_{k}, \boldsymbol{x}_{l}\right)=0$, then $(C, d)$ and $\left(C^{\prime}, d^{\prime}\right)$ are filtered chain homotopy equivalent.

Proof To prove that $\mathcal{F}^{\prime}$ defines a filtration on the reduced complex we must show

$$
\mathcal{F}^{\prime}\left(a, d^{\prime}(a)\right) \leq 0 \text { for all } a \in C^{\prime},
$$

which, by definition, is the same as showing

$$
\mathcal{F}(\iota(a)) \leq \mathcal{F}\left(\iota\left(d^{\prime}(a)\right)=\mathcal{F}(\iota \circ \pi \circ(d-d h d) \circ \iota(a)) .\right.
$$


To begin, observe that for any $x \in C$ we have $\mathcal{F}\left(\llcorner\circ \pi(x)) \geq \mathcal{F}(x)\right.$; that is, dropping $\boldsymbol{x}_{k}$ and $\boldsymbol{x}_{l}$ from a chain can only increase the filtration level. This is an immediate consequence of (7), and does not rely on homogeneity of $\boldsymbol{x}_{k}$ or $\boldsymbol{x}_{l}$. Indeed, for this lemma we do not assume that the filtration comes from a decomposition such as (6). Thus the right hand side of (8) satisfies

$$
\mathcal{F}(\iota \circ \pi \circ(d-d h d) \circ \iota(a)) \geq \mathcal{F}((d-d h d) \circ \iota(a)) .
$$

Let $r \in R$ denote $d\left(\iota(a), \boldsymbol{x}_{l}\right)$, the coefficient of $\boldsymbol{x}_{l}$ in $d(\iota(a))$. By the definition of $h$, the right hand of (9) is equal to $\mathcal{F}\left(d(\iota(a))-r \cdot d\left(\boldsymbol{x}_{k}\right)\right)$, which satisfies the inequality

$$
\mathcal{F}\left(d(\iota(a))-r \cdot d\left(x_{k}\right)\right) \geq \min \left\{\mathcal{F}\left(d(\iota(a)), \mathcal{F}\left(r d\left(\boldsymbol{x}_{k}\right)\right)\right\} .\right.
$$

Now if $\mathcal{F}\left(r d\left(\boldsymbol{x}_{k}\right)\right) \geq \mathcal{F}(d(\iota(a))$ then the right hand side of (10) equals $\mathcal{F}(d(\iota(a))$, which satisfies

$$
\mathcal{F}(d(\iota(a)) \geq \mathcal{F}(\iota(a)),
$$

because $(C, d)$ is filtered. If $\mathcal{F}\left(r d\left(x_{k}\right)\right)<\mathcal{F}(d(\iota(a))$, then $r \neq 0$ and the right side of (10) equals $\mathcal{F}\left(r d\left(x_{k}\right)\right)$ which satisfies $\mathcal{F}\left(r d\left(x_{k}\right)\right) \geq \mathcal{F}\left(\boldsymbol{x}_{l}\right)$ by assumption. In this case, though, we have

$$
\mathcal{F}\left(\boldsymbol{x}_{l}\right) \geq \mathcal{F}(\iota(a)),
$$

since $d\left(\iota(a), \boldsymbol{x}_{l}\right) \neq 0$ and $(C, d)$ is filtered. Thus in both cases, we have verified (8). The statement about filtration degree follows in essentially the same manner.

To see that $C$ and $C^{\prime}$ are filtered homotopy equivalent when the filtration levels of the cancelled chains agree, it suffices to show that the chain maps $f$ and $g$ and chain homotopy $h$ from the previous lemma are filtered, in the sense that

$$
\mathcal{F}^{\prime} \circ f \geq \mathcal{F}, \quad \mathcal{F} \circ g \geq \mathcal{F}^{\prime}, \quad \mathcal{F} \circ h \geq \mathcal{F} .
$$

These are immediate from the definition of $\mathcal{F}^{\prime}$ and the fact that $h$ respects $\mathcal{F}$ when $\mathcal{F}\left(\boldsymbol{x}_{k}, \boldsymbol{x}_{l}\right)=0$.

We can use the lemmas to easily produce a spectral sequence. To begin, define $\left(E_{0}, d_{0}\right)=(C, d)$. Now use Lemma 4.1 to cancel all of the nonzero terms in the differential of order zero ie the $d^{(0)}$ terms. Lemma 4.2 implies that the result is a filtered chain homotopy equivalent complex, $\left(E_{1}, d_{1}\right)$ for which the lowest order terms in the differential are of order one; that is, the differential can be written as $d_{1}=d_{1}^{(1)}+d_{1}^{(2)}+\cdots$ with respect to the natural direct sum decomposition of $E_{1}$ induced by (6). Now cancel the $d_{1}^{(1)}$ components of $d_{1}$. The result is a chain homotopy equivalent complex $\left(E_{2}, d_{2}\right)$ satisfying $d_{2}=d_{2}^{(2)}+d_{2}^{(3)}+\cdots$. Assuming the complex 
is finitely generated in each homological degree, we can iterate this process until all differentials of all orders have been cancelled. Denote the lowest order term of $d_{r}$ by

$$
\delta_{r}:=d_{r}^{(r)} .
$$

The fact that $d_{r} \circ d_{r}=0$ implies $\delta_{r} \circ \delta_{r}=0$, and canceling $d_{r}^{(r)}$ is equivalent to taking homology with respect to $\delta_{r}$. We represent the process schematically:

$$
\begin{array}{cc}
E_{0}, d_{0}=d^{(0)}+d^{(1)}+d^{(2)}+d^{(3)}+\cdots & \\
\downarrow & \text { Homology with respect to } \delta_{0}=d^{(0)} \\
E_{1}, d_{1}=0+d_{1}^{(1)}+d_{1}^{(2)}+d_{1}^{(3)}+\cdots & \\
\downarrow & \text { Homology with respect to } \delta_{1}=d_{1}^{(1)} \\
E_{2}, d_{2}=0+0+d_{2}^{(2)}+d_{2}^{(3)}+\cdots & \\
\downarrow & \text { Homology with respect to } \delta_{2}=d_{2}^{(2)} \\
\vdots & \\
E_{\infty}, d_{\infty} \equiv 0 &
\end{array}
$$

The resulting structure is a spectral sequence with $r^{\text {th }}$ page given by $\left(E_{r}, \delta_{r}\right)$. By construction, $E_{\infty} \cong H_{*}(C, d)$, since $\left(E_{\infty}, d_{\infty}\right)$ is chain homotopy equivalent to $(C, d)$. The concerned reader can take comfort in the knowledge that the spectral sequence described here is isomorphic to the one which is produced by the standard construction; see [20, Theorem 2.6]. The proof of equivalence is straightforward but rather notationally cumbersome, and since our results make no use of it we leave it for the interested reader.

Let $C$ and $\bar{C}$ be complexes with filtrations induced by decompositions

$$
C=\bigoplus_{i \geq 0} C^{(i)} \bar{C}=\bigoplus_{i \geq 0} \bar{C}^{(i)}
$$

and let $a: C \rightarrow \bar{C}$ be a filtered chain map, ie $a\left(F^{i}\right) \subset \bar{F}^{i}$. Such a map is well-known to induce a morphism between the associated spectral sequences; that is a sequence of chain maps

$$
\alpha_{r}:\left(E_{r}, \delta_{r}\right) \longrightarrow\left(\bar{E}_{r}, \bar{\delta}_{r}\right) \quad \text { satisfying }\left(\alpha_{r}\right)_{*}=\alpha_{r+1} .
$$

A standard treatment of this construction can be found in [20, pages 65-67]. The perspective of reduction offers a concrete construction of this morphism as follows. To begin, note that a filtered chain map decomposes into homogeneous summands in a manner similar to the differential

$$
a=a^{(0)}+a^{(1)}+a^{(2)}+\cdots, \quad \text { where } a^{(m)}: C^{(i)} \rightarrow \bar{C}^{(i+m)} .
$$


Recalling that $E_{0}=C$ and $\bar{E}_{0}=\bar{C}$, let $a_{0}: E_{0} \rightarrow \bar{E}_{0}$ be defined as $a_{0}:=a$. Let

$$
g_{r}:\left(E_{r}, d_{r}\right) \rightarrow\left(E_{r+1}, d_{r+1}\right), \quad \bar{g}_{r}:\left(\bar{E}_{r}, \bar{d}_{r}\right) \rightarrow\left(\bar{E}_{r+1}, \bar{d}_{r+1}\right),
$$

denote the chain homotopy equivalences that cancel the $r^{\text {th }}$ order terms in the differential, and inductively define $a_{r+1}:\left(E_{r+1}, d_{r+1}\right) \rightarrow\left(\bar{E}_{r+1}, \bar{d}_{r+1}\right)$ by

$$
a_{r+1}=\bar{g}_{r} \circ a_{r} \circ\left(g_{r}\right)^{-1},
$$

where $\left(g_{r}\right)^{-1}$ is the chain homotopy inverse of $g_{r}$. Now $a_{r}$ decomposes into summands according to the filtration, and it is easy to see that each $a_{r}$ respects the filtration, ie

$$
a_{r}=a_{r}^{(0)}+a_{r}^{(1)}+a_{r}^{(2)}+\cdots .
$$

Indeed, this holds by assumption for $a_{0}=a$, and the homotopy equivalences $\left(g_{r}\right)^{-1}$ and $\bar{g}_{r}$ provided by the cancellation lemma are all nondecreasing in filtration degree (they involve terms of the form $h \circ d_{r}$ and $\bar{d}_{r} \circ \bar{h}$, respectively, where $h, \bar{h}$ have degree $-r$ and $d_{r}, \bar{d}_{r}$ have degree $r$.) Let the $0^{\text {th }}$ order term of $a_{r}$ be denoted by

$$
\alpha_{r}:=a_{r}^{(0)} .
$$

The $0^{\text {th }}$ order terms of the equality $a_{r} \circ d_{r}=\bar{d}_{r} \circ a_{r}$ must themselves be equal, which implies $\alpha_{r} \circ \delta_{r}=\bar{\delta}_{r} \circ \alpha_{r}$. Thus $\alpha_{r}$ is a chain map between $\left(E_{r}, \delta_{r}\right)$ and $\left(\bar{E}_{r}, \bar{\delta}_{r}\right)$, and by the definition of the map induced on homology, we have $\left(\alpha_{r}\right)_{*}=\alpha_{r+1}$. This provides the desired morphism of spectral sequences.

It should be pointed out that one can verify that the morphism constructed above agrees with the more traditional construction. Since we will not make use of this fact we again omit the proof. We should also point out that in many situations, one or all of the intermediate chain maps $a_{r}$ may have vanishing $0^{\text {th }}$ order terms; that is $a_{r}^{(0)}=0$. The same rational which shows $a_{r}^{(0)}=\alpha_{r}$ is a chain map shows that the lowest order nonvanishing term is a chain map. This allows one to construct a morphism of spectral sequences where the filtration shift of the maps between successive pages is monotonically increasing with the page index.

\subsection{An action on the link surgeries spectral sequence}

Let $L=K_{1} \cup \cdots \cup K_{l} \subset Y$ be a framed link. In this subsection we prove that a curve $\gamma \subset Y \backslash L$ gives rise to a filtered endomorphism of the filtered complex that produces the link surgeries spectral sequence. To state the theorem, we establish a bit of notation. A multiframing is a $l$-tuple $I=\left\{m_{1}, \ldots, m_{l}\right\} \in \widehat{\mathbb{Z}}^{l}$, where $\widehat{\mathbb{Z}}=\mathbb{Z} \cup \infty$. A multiframing specifies a $3-$ manifold, which we denote $Y(I)$, by performing surgery 
on $L$ with slope on the $i^{\text {th }}$ component given by $m_{i}$ (defined with respect to the base framing). Thus $Y(\infty, \ldots, \infty)=Y$ corresponds to not doing surgery at all.

Theorem 4.3 Let $L \subset Y$ be a framed link. There is a filtered complex $(C(L), d)$ whose homology is isomorphic to $\widehat{H F}(Y)$. The $E_{1}$ page of the associated spectral sequence satisfies

$$
E_{1} \cong \bigoplus_{I \in\{0,1\}|L|} \widehat{H F}(Y(I))
$$

A curve $\zeta \subset Y \backslash L$ induces a filtered chain map

$$
a^{\zeta}: C(L) \longrightarrow C(L)
$$

whose induced map is isomorphic to the action

$$
\left(a^{\zeta}\right)_{*}=A^{[\zeta]}: \widehat{H F}(Y) \longrightarrow \widehat{H F}(Y) .
$$

The induced map on the $E_{1}$ page of the spectral sequence is given by the sum

$$
\bigoplus_{I} \widehat{H F}(Y(I)) \stackrel{\oplus_{I} A_{I}^{[\zeta]}}{\longrightarrow} \bigoplus_{I} \widehat{H F}(Y(I)),
$$

where $A_{I}^{[\zeta]}$ is the map on $\widehat{H F}(Y(I))$ induced by $\zeta$, viewed as a curve in $Y(I)$.

Proof The existence of the filtered complex computing $\widehat{H F}(Y)$ is [28, Theorem 4.1]. The refinement here is the existence of a filtered chain map associated to a curve in the framed link diagram. This map will be defined as a sum of the holomorphic polygon operators $a_{0,1, \ldots, n}^{\zeta}$ from Section 3.2. In order to make this precise, we must recall the proof of Ozsváth and Szabó's theorem. There are essentially two main steps. The first is to construct a filtered chain complex $(X, D)$ from a framed link $L \subset Y$, and the second is to show that a natural (filtered) subcomplex $(C(L), d)$ has homology isomorphic to $\widehat{H F}(Y)$. In both steps, we will take care to introduce our refinement at the appropriate time.

The first step in the proof is to associate a filtered complex to a Heegaard multidiagram adapted to a framed link $L=K_{1} \cup \cdots \cup K_{l} \subset Y$. More precisely, we have a Heegaard multidiagram

$$
\left(\Sigma, \eta^{0}, \eta^{1}, \ldots, \eta^{k}, w\right)
$$

with the property that the 3 -manifolds $Y_{0, i}(i=1, \ldots, k)$ are in one-to-one correspondence with the 3 -manifolds $Y(I)$ associated to all possible multiframings $I=\left\{m_{1}, \ldots, m_{l}\right\}$, with $m_{i} \in\{0,1, \infty\}$. The 3 -manifolds $Y_{i, j}$ for $i, j>0$ are all 
diffeomorphic to the connected sum of a number of copes of $S^{1} \times S^{2}$. As usual, we require the multidiagram to be admissible.

From such a Heegaard diagram one constructs a filtered complex $(X, D)$. As a group, the complex splits as a direct sum over the set of multiframings:

$$
X=\bigoplus_{I \in\{0,1, \infty\}^{l}} \widehat{C F}(Y(I))
$$

The differential on $\mathrm{X}$ is given as a sum of holomorphic polygon maps associated to certain submultidiagrams. Call a multiframing $J$ an immediate successor of a multiframing $I$ if the two framings differ on exactly one component $K_{i} \subset L$ and if the restriction of $J$ to $K_{i}$ is one greater than the restriction of $I$, taken with respect to the length two ordering $0<1<\infty$. Write $I<J$ if $J$ is an immediate successor of $I$. Given any sequence of immediate successors $I^{0}<I^{1}<\cdots<I^{m}$, there is a map

$$
D_{I^{0}<\cdots<I^{m}}: \widehat{C F}\left(Y\left(I^{0}\right)\right) \longrightarrow \widehat{C F}\left(Y\left(I^{m}\right)\right)
$$

defined by the pseudoholomorphic polygon map of Section 3.2

$$
D_{I^{0}<\cdots<I^{m}}(-):=f_{0, i_{0}, \ldots, i_{m}}\left(-\otimes \Theta_{1} \otimes \cdots \otimes \Theta_{m}\right) .
$$

The indexing $\left(0, i_{0}, \ldots, i_{m}\right)$ which specifies the submultidiagram

$$
\left(\Sigma, \eta^{0}, \eta^{i_{0}}, \ldots, \eta^{i_{m}}, w\right)
$$

is determined by the requirement that $Y_{0, i_{j}}=Y\left(I^{j}\right)$ for each $j=0,1, \ldots, m$, under the bijection between the 3-manifolds $Y_{0, i}$ and those associated to the multiframings. Here and throughout, $\Theta_{j}$ is a cycle generating the highest graded component of $\widehat{H F}_{*}\left(Y_{i_{j-1}, i_{j}}\right)$. For a sequence of successors of length zero, we have that $D_{I}: \widehat{C F}(Y(I)) \rightarrow \widehat{C F}(Y(I))$ is simply the Floer differential.

Ozsváth and Szabó define an endomorphism $D: X \rightarrow X$ by

$$
D=\sum_{\left\{I^{0}<I^{1}<\cdots<I^{m}\right\}} D_{I^{0}<I^{1}<\cdots<I^{m}},
$$

where the sum is over all sequences of immediate successors (of all lengths). They show that $D \circ D=0$ [28, Proposition 4.4]. The key tool in their proof is the $A_{\infty}$ relation for pseudoholomorphic polygon maps, together with a vanishing theorem for these maps in the present context, [28, Lemma 4.2].

The resulting complex $(X, D)$ has a natural filtration by the totally ordered set $\{0,1, \infty\}^{l}$ which arises from its defining decomposition along multiframings. We can also collapse 
this to a $\mathbb{Z}$-filtration, and it is this latter filtration which will be of primary interest. To do this, let the norm of a multiframing $I=\left(m_{1}, \ldots, m_{l}\right)$ be given by

$$
|I|=\sum_{i=1}^{l} m_{i}^{\prime},
$$

where $m_{i}^{\prime}=m_{i}$ if $m_{i} \in\{0,1\}$ and $m_{i}^{\prime}=2$ if $m_{i}=\infty$. Then

$$
X=\bigoplus_{i \geq 0} C^{(i)}
$$

where

$$
C^{(i)}=\bigoplus_{\left\{I \in\{0,1, \infty\}^{l}|| I \mid=i\right\}} \widehat{C F}(Y(I)) .
$$

The differential $D$ clearly respects the decomposition, giving rise to a decreasing filtration of $X$ by subcomplexes $F^{p}=\oplus_{i \geq p} C^{(i)}$, as in the previous subsection. The $0^{\text {th }}$ page of the spectral sequence is given by

$$
E_{0}=\bigoplus_{I \subset\{0,1, \infty\}^{l}} \widehat{C F}(Y(I))
$$

with $\delta_{0}$ differential given simply as the Floer differential; that is, $\left.\delta_{0}\right|_{\widehat{C F}(Y(I))}=D_{I}$. The $E_{1}$ page of the spectral sequence splits as

$$
E_{1} \cong \bigoplus_{I} \widehat{H F}(Y(I))
$$

with $\left.\delta_{1}\right|_{\hat{H F}(Y(I))}$ given by $\sum\left(D_{I<J}\right)_{*}$, the sum over all immediate successors of $I$, of the maps induced on homology by $D_{I<J}$. These are the maps

$$
\widehat{F}_{W}: \widehat{H F}(Y(I)) \longrightarrow \widehat{H F}(Y(J))
$$

associated to the 2-handle cobordism between $Y(I)$ and $Y(J)$ (here we are using the fact that $J$ is an immediate successor of $I$ ).

We are now in a position to understand the action of a curve $\zeta \subset Y \backslash L$. Without loss of generality, we may assume that $\zeta$ lies on the Heegaard surface, and is in general position with all attaching curves. Define maps

$$
a_{I^{0}<\cdots<I^{m}}^{\zeta}: \widehat{C F}\left(Y\left(I^{0}\right)\right) \longrightarrow \widehat{C F}\left(Y\left(I^{m}\right)\right)
$$

by the polygon operators from Section 3.2,

$$
a_{I^{0}<\cdots<I^{m}}^{\zeta}(-):=a_{0, i_{0}, \ldots, i_{m}}^{\zeta}\left(-\otimes \Theta_{1} \otimes \cdots \otimes \Theta_{m}\right) .
$$


The sum of all these maps (over all sequences of immediate successors) is an endomorphism $a^{\zeta}: X \rightarrow X$, which the following lemma shows is a chain map. This map forms the basis of our refinement.

Lemma 4.4 The map $a^{\zeta}: X \rightarrow X$ given by

$$
a^{\zeta}=\sum_{\left\{I^{0}<I^{1}<\cdots<I^{m}\right\}} a_{I^{0}<I^{1}<\cdots<I^{m}}^{\zeta}
$$

is a chain map. Moreover, $a^{\zeta}$ respects the collapsed filtration $F^{i}$ and the filtration of $(X, D)$ by the totally ordered set $\{0,1, \infty\}^{l}$.

Proof We want to prove $D \circ a^{\xi}+a^{\xi} \circ D=0$. The proof is analogous to [28, Proposition 4.4]. Given $I, J$ such that $|I|<|J|$, we expand the component map

$$
D \circ a^{\zeta}+a^{\zeta} \circ D: \widehat{C F}(Y(I)) \longrightarrow \widehat{C F}(Y(J))
$$

to get

$$
\sum_{I=I^{0}<\cdots<I^{m}=J} \sum_{0 \leq r \leq m}\left(D_{I^{r}<\cdots<I^{m}} \circ a_{I^{0}<\cdots<I^{r}}^{\zeta}+a_{I^{r}<\cdots<I^{m}}^{\zeta} \circ D_{I^{0}<\cdots<I^{r}}\right),
$$

where the first sum is over all sequences of immediate successors connecting $I$ to $J$. Such sequences must be of length $m=|J|-|I|$. Pick one of these sequences $I^{0}<\cdots<I^{m}$, and consider the term on the left in the second summation, applied to a chain $\boldsymbol{x}$

$$
\begin{aligned}
\sum_{0 \leq r \leq m} D_{I^{r}}<\cdots<I^{m} \circ a_{I^{0}<\cdots<I^{r}}^{\zeta} & (x) \\
& :=\sum_{0 \leq r \leq m} f_{0, i_{r}, \ldots, i_{m}}\left(a_{0, i_{0}, \ldots, i_{r}}^{\zeta}\left(x \otimes \Theta_{1} \otimes \cdots \otimes \Theta_{r}\right) \otimes \Theta_{r+1} \otimes \cdots \otimes \Theta_{m}\right),
\end{aligned}
$$

where the indices $i_{n}$ refer to the sets of attaching curves for which $Y_{0, i_{n}} \simeq Y\left(I^{n}\right)$, as above. Theorem 3.4 indicates that this is equal to

$$
\begin{aligned}
\sum_{0 \leq r<s \leq m} a_{0, i_{0}, \ldots, i_{r}, i_{s}, \ldots, i_{m}}\left(\boldsymbol{x} \otimes \cdots \otimes f_{i_{r}, \ldots, i_{s}}\left(\Theta_{r+1} \otimes \cdots \otimes \Theta_{s}\right) \otimes \cdots \otimes \Theta_{m}\right) \\
+\sum_{0 \leq s \leq m} a_{0, i_{s}, \ldots, i_{m}}^{\zeta}\left(f_{0, i_{0}, \ldots, i_{s}}\left(\boldsymbol{x} \otimes \cdots \otimes \Theta_{s}\right) \otimes \Theta_{s+1} \otimes \cdots \otimes \Theta_{m}\right) .
\end{aligned}
$$

But the second term in the above sum is equal to, and hence cancels over $\mathbb{F}$, the $a^{\zeta} \circ D$ term from the inner sum in (11). Thus (11) becomes

$\sum_{I^{0}<\cdots<I^{m}} \sum_{0 \leq r<s \leq m} a_{0, i_{0}, \ldots, i_{r}, i_{s}, \ldots, i_{m}}^{\zeta}\left(x \otimes \cdots \otimes f_{i_{r}, \ldots, i_{s}}\left(\Theta_{r+1} \otimes \cdots \otimes \Theta_{s}\right) \otimes \cdots \otimes \Theta_{m}\right)$. 
This expression can be rewritten as

$$
\sum_{\substack{I^{\prime}=: I^{r} \\ J^{\prime}=: I^{s}}} \sum_{\substack{I^{0}<\cdots<I^{r} \\ I^{s}<\cdots<I^{m}}} a_{0, i_{0}, \ldots, i_{r}, i_{s}, \ldots, i_{m}}^{\zeta}\left(\boldsymbol{x} \otimes \cdots \otimes \sum_{I^{r}<\cdots<I^{s}} f_{i_{r}, \ldots, i_{s}}\left(\Theta_{r+1} \otimes \cdots \otimes \Theta_{S}\right) \otimes \cdots \otimes \Theta_{m}\right),
$$

where $I^{\prime}$ and $J^{\prime}$ in the first sum range over all multiframings between $I$ and $J$ (which we subsequently relabel $I^{\prime}=I^{r}$ and $J^{\prime}=I^{s}$ ). The second sum ranges over all sequences of successors connecting $I$ to $I^{\prime}$ and $J^{\prime}$ to $J$. The third sum ranges over all sequences of successors connecting $I^{\prime}$ to $J^{\prime}$. However, [28, Lemma 4.3] states that $\sum_{I^{r}<\cdots<I^{s}} f_{i_{r}, \ldots, i_{s}}\left(\Theta_{r+1} \otimes \cdots \otimes \Theta_{s}\right) \equiv 0$, for any $I^{r}$ and $I^{s}$. This shows that $a^{\zeta}$ is a chain map. It clearly respects the filtration $F^{i}$ (since it splits into homogeneous summands by the length of the sequence of successors) and the subcomplexes of the filtration of $(X, D)$ by the totally ordered set $\{0,1, \infty\}^{l}$.

The second step in the proof of the Ozsváth-Szabó theorem is to show that $(X, D)$ has a natural quotient complex $(C(L), d)$ whose homology is isomorphic to $\widehat{H F}(Y)$. Given a subset of multiframings $S \subset\{0,1, \infty\}^{l}$, let $X(S)$ denote the subgroup of $X$ given by

$$
X(S)=\bigoplus_{I \in S} \widehat{C F}(Y(I))
$$

Let $(C(L), d)=X\left(\{0,1\}^{l}\right)$ be the group generated by all multiframings which do not contain $\infty$. This is clearly a quotient complex, with associated subcomplex generated by those multiframings which contain $\infty$. The complex $X\left(\{0,1\}^{l}\right)$ inherits the filtration from $X$, and the curve map $a^{\xi}$ induces a filtered chain map from $X\left(\{0,1\}^{l}\right)$ to itself. Ozsváth and Szabó show that the homology of $(C(L), d)$ is isomorphic to $\widehat{H F}(Y)$, and our task is to show that their isomorphism fits into the diagram

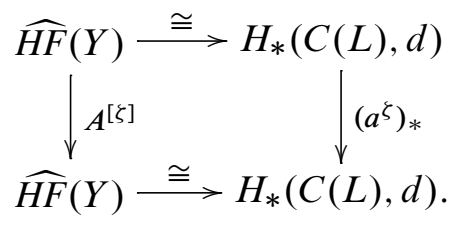

The key ingredient is a refined version of the strong form of the surgery exact triangle; see [28, Theorem 4.5]. Let $K$ be a framed knot in a 3-manifold $Y$, and let

$$
f=D_{0<1}: \widehat{C F}(Y(0)) \longrightarrow \widehat{C F}(Y(1))
$$

be the map induced by the 2-handle cobordism. Ozsváth and Szabó [28, Theorem 4.5] show that the mapping cone complex $M(f)$ is quasi-isomorphic to $\widehat{C F}(Y)$. We must 
account for the $\Lambda^{*}\left(H_{1}(Y ; \mathbb{Z}) /\right.$ Tors $)$ action. To do this let $\zeta \subset Y \backslash K$ be a curve, as usual. Consider the complex

$$
X(\{0,1, \infty\})=\widehat{C F}(Y(0)) \oplus \widehat{C F}(Y(1)) \oplus \widehat{C F}(Y(\infty)),
$$

endowed with the differential

$$
D=\left(\begin{array}{ccc}
D_{0} & 0 & 0 \\
D_{0<1} & D_{1} & 0 \\
D_{0<1<\infty} & D_{1<\infty} & D_{\infty}
\end{array}\right)
$$

There is a natural short exact sequence

$$
0 \longrightarrow X(\{\infty\}) \longrightarrow X(\{0,1, \infty\}) \longrightarrow X(\{0,1\}) \longrightarrow 0
$$

where the sub and quotient complexes are identified with $\widehat{C F}(Y)$ and $M(f)$, respectively. Ozsváth and Szabó show that $X(\{0,1, \infty\})$ is acyclic [28, Lemma 4.2], proving that $M(f)$ is quasi-isomorphic to $\widehat{C F}(Y)$.

We have the map

$$
a^{\zeta}: X(\{0,1, \infty\}) \longrightarrow X(\{0,1, \infty\})
$$

given by

$$
a^{\zeta}=\left(\begin{array}{ccc}
a_{0}^{\zeta} & 0 & 0 \\
a_{0<1}^{\zeta} & a_{1}^{\zeta} & 0 \\
a_{0<1<\infty}^{\zeta} & a_{1<\infty}^{\zeta} & a_{\infty}^{\zeta}
\end{array}\right),
$$

which Lemma 4.4 shows is a chain map. Moreover, $a^{\xi}$ respects the short exact sequence (12), thus inducing a map on the sub and quotient complex. The map on the subcomplex is simply $a_{\infty}^{\zeta}: \widehat{C F}\left(Y_{\infty}\right) \rightarrow \widehat{C F}\left(Y_{\infty}\right)$ and the map on the quotient complex is

$$
a_{M(f)}^{\zeta}=\left(\begin{array}{cc}
a_{0}^{\zeta} & 0 \\
a_{0<1}^{\zeta} & a_{1}^{\zeta}
\end{array}\right): M(f) \longrightarrow M(f) .
$$

Considering the corresponding long exact sequence in homology, we obtain a commutative diagram

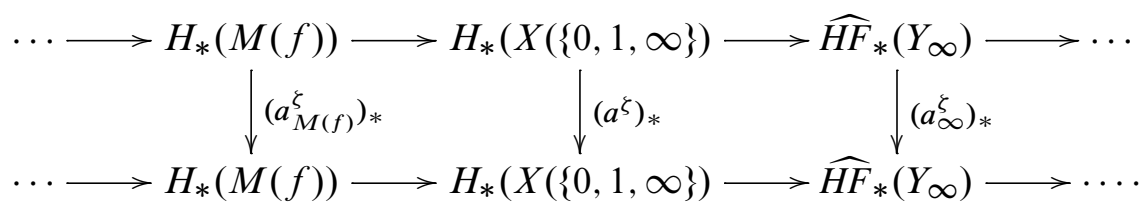


By Ozsváth and Szabó's theorem, $H_{*}(X(\{0,1, \infty\})=0$, showing that

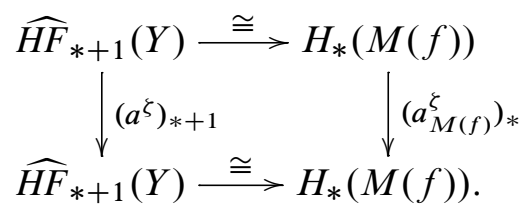

This can be interpreted as saying that the Floer homology of $Y$ is isomorphic to the mapping cone of the 2 -handle map, as a module over $\Lambda^{*}\left(H_{1}(Y ; \mathbb{Z}) /\right.$ Tors $)$.

Given this refinement of the surgery exact triangle, the proof of Theorem 4.3 proceeds quickly by the same inductive argument used in the proof of [28, Theorem 4.1]. Specifically, we return to the complex $X$ coming from a framed link diagram of $l$ components. If $l=1$, the preceding discussion proves the theorem, showing that the filtered complex $X(\{0,1\})$ computes the homology of $X(\infty)=\widehat{C F}(Y)$, and that a curve $\zeta \subset Y \backslash K$ induces a filtered chain map whose induced map agrees with that of $a^{\zeta}: \widehat{C F}(Y) \rightarrow \widehat{C F}(Y)$. Assume that this remains true for the complex $X\left(\{0,1\}^{l-1}\right)$ associated to an $(l-1)$-component link and the induced map $a^{\zeta}$. That is, we have a commutative diagram

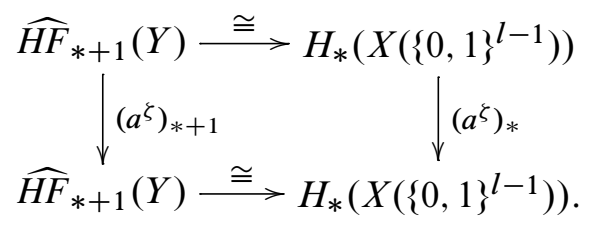

Turn now to an $l$-component link and consider the complex $X\left(\{0,1\}^{l-1} \times\{0,1, \infty\}\right)$ (that this is a complex follows from the fact that it is a quotient of $X$ by the subcomplex consisting of multiframings with at least one of the first $l-1$ parameters equals to $\infty$ ). There is the short exact sequence, compatible with the maps induced by $a^{\xi}$ :

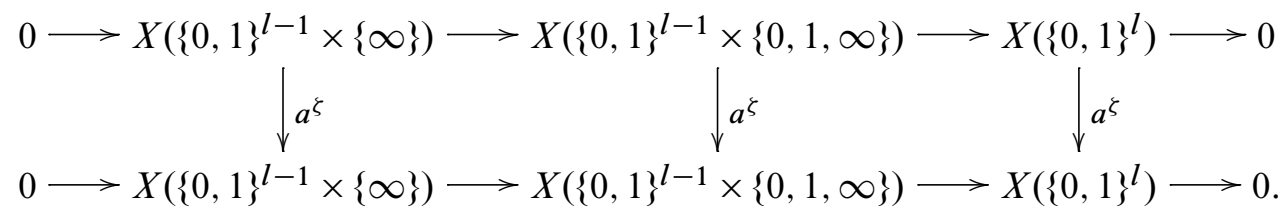

The middle term has a natural filtration coming from the total ordering on $\{0,1\}^{l-1}$. The associated graded groups of this filtration are each isomorphic to $H_{*}(X(I \times\{0,1, \infty\}))$ for some multiframing $I \subset\{0,1\}^{l-1}$. The strong form of the surgery exact triangle, however, implies that these groups are all zero. Thus, $H_{*}\left(X\left(\{0,1\}^{l-1} \times\{0,1, \infty\}\right)\right)=0$. 
Thus we have the diagram

$$
\begin{gathered}
H_{*+1}\left(X\left(\{0,1\}^{l-1} \times\{\infty\}\right)\right) \stackrel{\cong}{\longrightarrow} H_{*}\left(X\left(\{0,1\}^{l}\right)\right) \\
\downarrow\left(a^{\zeta}\right)_{*+1}\left(a^{\zeta}\right)_{*} \\
H_{*+1}\left(X\left(\{0,1\}^{l-1} \times\{\infty\}\right)\right) \stackrel{\cong}{\longrightarrow} H_{*}\left(X\left(\{0,1\}^{l}\right)\right) .
\end{gathered}
$$

Our inductive hypothesis equates the left hand side with $A^{[\zeta]}: \widehat{H F}(Y) \rightarrow \widehat{H F}(Y)$. This completes the proof that the map induced on homology by $a^{\zeta}$ is isomorphic to $A^{[\zeta]}$.

To see that the induced map $\alpha_{1}^{\zeta}$ on the $E_{1}$ page is given by

$$
\bigoplus_{I} \widehat{H F}(Y(I)) \stackrel{\oplus_{I} A_{I}^{[\zeta]}}{\longrightarrow} \bigoplus_{I} \widehat{H F}(Y(I)),
$$

it suffices to recall that $\alpha_{1}^{\zeta}$ is the induced map on the homology of $\left(E_{0}, \delta_{0}\right)$ by $\alpha_{0}^{\zeta}$, where $\alpha_{0}^{\zeta}$ is the map on the $E_{0}$ page induced by $a^{\zeta}$. But $\alpha_{0}^{\zeta}$, in turn, is simply the lowest order term of $a^{\zeta}$ and is given by

$$
\bigoplus_{I} \widehat{C F}(Y(I)) \stackrel{\oplus_{I} a_{I}^{\zeta}}{\longrightarrow} \bigoplus_{I} \widehat{C F}(Y(I)),
$$

where $a_{I}^{\zeta}: \widehat{C F}(Y(I)) \rightarrow \widehat{C F}(Y(I))$ is the operator obtained by viewing $\zeta$ as a curve in $Y(I)$. By definition, we have $A_{I}^{[\zeta]}=\left(a_{I}^{\zeta}\right)_{*}$.

\subsection{Connecting the Khovanov module to the Floer module}

Let

$$
\Lambda_{n-1}^{*}=\mathbb{F}\left[X_{1}, \ldots, X_{n-1}\right] /\left(X_{1}^{2}, \ldots, X_{n-1}^{2}\right) .
$$

As discussed in Section 2, a marked point $p_{0}$ on one component of a link diagram gives rise to the reduced Khovanov chain complex, $C K h^{r}$. Choosing a marked point on each remaining component gives the reduced Khovanov homology $\Lambda_{n-1}^{*}$-module structure. More precisely, additional marked points give rise to chain maps

$$
x_{i}: C K h^{r}(L) \longrightarrow C K h^{r}(L), \quad i=1, \ldots, n-1,
$$

which satisfy $x_{i} \circ x_{j}=x_{j} \circ x_{i}$ and $x_{i} \circ x_{i}=0$.

Consider a properly embedded arc $t_{i} \subset\left(S^{3}, L\right)$ connecting $p_{0}$ to the $i^{\text {th }}$ additional marked point. The preimage of this arc in the branched double cover is a closed curve $\zeta_{i}=\pi^{-1}\left(t_{i}\right) \subset \Sigma(L)$. As in Section 3.1, we can assume that $\zeta_{i}$ lies on the Heegaard 
surface of a Heegaard diagram of $\Sigma(L)$, and hence we obtain a chain map on the associated Floer complex,

$$
a^{\zeta_{i}}: \widehat{C F}(\Sigma(L)) \longrightarrow \widehat{C F}(\Sigma(L)) .
$$

This chain map is related to the chain map $x_{i}$ on the Khovanov complex by the following theorem.

Theorem 4.5 Let $D$ be a diagram for a link $L=K_{0} \cup \cdots \cup K_{n-1} \subset S^{3}$, together with a base point $p_{0} \subset K_{0}$. There is a filtered chain complex, $(C(D), d)$, whose homology is isomorphic to $\widehat{H F}(\Sigma(L))$. The associated spectral sequence satisfies

$$
\left(E_{1}, \delta_{1}\right) \cong\left(C K h^{r}(\bar{D}), \partial\right),
$$

where $\bar{D}$ is the mirror of $D$ and the reduced Khovanov complex is defined with $p_{0}$. Let $t_{i}$ be a proper arc connecting $p_{0} \subset K_{0}$ to $p_{i} \subset K_{i}$, and $\zeta_{i}=\pi^{-1}\left(t_{i}\right) \subset \Sigma(L)$ its lift to the branched double cover. Then there is a filtered chain map

$$
a^{\zeta_{i}}:(C(D), d) \longrightarrow(C(D), d),
$$

whose induced map on homology satisfies

$$
\left(a^{\zeta_{i}}\right)_{*}=A^{\left[\zeta_{i}\right]}: \widehat{H F}(\Sigma(L)) \longrightarrow \widehat{H F}(\Sigma(L)) .
$$

The induced map $\alpha_{1}^{\zeta_{i}}$ on the $E_{1}$ page of the spectral sequence satisfies

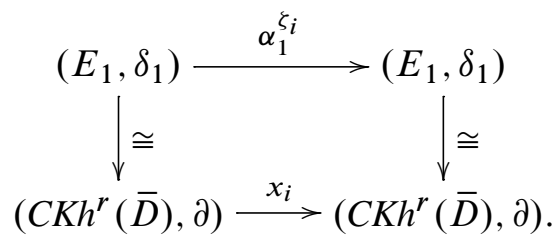

Proof The first part of the theorem is the content of [28, Theorem 1.1], which is a rather immediate consequence of the link surgeries spectral sequence, applied to a particular surgery presentation of $\Sigma(L)$ coming from the link diagram. The key point is that the branched double covers of links which differ by the unoriented skein relation differ by a triad of surgeries along a framed knot in that manifold. Moreover, the branched double cover of the natural saddle cobordism passing between the zero and one resolution is the 2-handle cobordism between the branched double covers which appears in the surgery exact triangle. The branched double cover of a complete resolution is diffeomorphic to $\#^{k} S^{1} \times S^{2}$, where $k+1$ is the number of components of the resolution, and the Heegaard Floer homology of $\#^{k} S^{1} \times S^{2}$ is isomorphic to the reduced Khovanov homology of the complete resolution. Moreover, the maps between the Floer homology of the connected sums of copies of $S^{1} \times S^{2}$ induced 
by the 2 -handle cobordisms agree with the Frobenius algebra defining the reduced Khovanov differential.

In the present situation, we wish to keep track of an action. In the case of Khovanov homology it is the action $x_{i}$ induced by a point $p_{i} \in K_{i}$, whereas in the Floer setting it is the action of the curve $\zeta_{i}=\pi^{-1}\left(t_{i}\right)$ arising as the lift of an arc $t_{i}$ connecting $p_{0}$ to $p_{i}$. Theorem 4.3 shows that $\zeta_{i}$ induces a filtered chain map

$$
a^{\zeta_{i}}: X\left(\{0,1\}^{l}\right) \rightarrow X\left(\{0,1\}^{l}\right),
$$

whose induced map on homology agrees with $A^{\left[\zeta_{i}\right]}: \widehat{H F}(\Sigma(L)) \rightarrow \widehat{H F}(\Sigma(L))$. Thus it suffices to see that the induced map on the $E_{1}$ page of the spectral sequence agrees with the Khovanov action. This follows from the fact that the induced map $\alpha_{1}^{\zeta_{i}}:\left(E_{1}, \delta_{1}\right) \rightarrow\left(E_{1}, \delta_{1}\right)$ is simply the sum of the actions of $\zeta_{i}$ on the Floer homology groups at each individual vertex in the cube; that is,

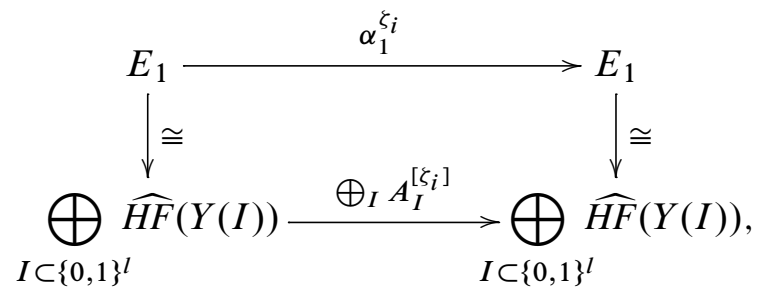

where $A_{I}^{\left[\zeta_{i}\right]}: \widehat{H F}(Y(I)) \rightarrow \widehat{H F}(Y(I))$ is the map induced by $\zeta_{i}$, viewed as a curve in $Y(I)$. Now we have each $Y(I)$ is diffeomorphic to $\#^{k} S^{1} \times S^{2}$ for some $k$, and

$$
\widehat{H F}\left(\stackrel{k}{\#} S^{1} \times S^{2}\right) \cong \mathbb{F}\left[x_{1}, \ldots, x_{k}\right] /\left(x_{1}^{2}, \ldots, x_{k}^{2}\right)
$$

as a module over $\Lambda^{*}\left(H_{1}\left(\#^{k} S^{1} \times S^{2}\right)\right)$. Here a basis for $H_{1}\left(\#^{k} S^{1} \times S^{2}\right)$ is identified with the variables $x_{1}, \ldots, x_{k}$, and such a basis is given by the lift of any $k$ proper arcs connecting the unknot containing $p_{0}$ to each of the $k$ other unknots. Under this correspondence, the action of $\zeta_{i}$ on the Floer homology of the branched double cover of a complete resolution agrees with the action of $x_{i}$ on the reduced Khovanov homology of the complete resolution. This completes the proof.

The preceding theorem allows us to endow the entire Ozsváth-Szabó spectral sequence with a module structure. To describe this, we say that a ring $R$ acts on a spectral sequence $\left\{\left(E_{i}, \delta_{i}\right)\right\}_{i=0}^{\infty}$ if for each $i$ we have: 
(1) $E_{i}$ is an $R$-module.

(2) The differential is $R$-linear: $x \cdot \delta_{i}(\beta)=\delta_{i}(x \cdot \beta)$ for all $x \in R, \beta \in E_{i}$; Equivalently, $R$ acts on $E_{i}$ by chain maps.

(3) The $R$-module structure on $E_{i+1}$ is induced through homology by the module structure on $E_{i}$.

If the above conditions hold only for $i \geq r$, we say that the action begins at the $r^{\text {th }}$ page. The following is now an easy corollary of Theorem 4.5.

Corollary 4.6 Let $L=K_{0} \cup \cdots \cup K_{n-1} \subset S^{3}$ be a link. Then the spectral sequence from the reduced Khovanov homology of $\bar{L}$ to the Floer homology of $\Sigma(L)$ is acted on by $\Lambda_{n-1}^{*}$. The resulting module structure on Khovanov homology is isomorphic to the module structure induced by the basepoint maps.

Remark Reduced Khovanov homology appears as the $E_{2}$ page of a spectral sequence. As our proof indicates, the action begins at $E_{1}$. The action on $E_{0}$ holds only up to chain homotopy.

Proof For each component $K_{i} \subset L$, choose a basepoint $p_{i} \subset K_{i}$ on a diagram for $L$. Pick a system of arcs $t_{i}$ connecting $p_{0}$ to $p_{i}$, and consider the closed curves $\zeta_{i}=\pi^{-1}\left(t_{i}\right), i=1, \ldots, n-1$, arising from their lifts to $\Sigma(L)$. According to Theorem 4.5 we obtain a collection of filtered chain maps,

$$
a^{\zeta_{i}}:(C, d) \longrightarrow(C, d), \quad i=1, \ldots, n-1,
$$

acting on the filtered chain complex which gives rise to the Ozsváth-Szabó spectral sequence.

Consider the free group $F_{n-1}$ on $n-1$ generators, $X_{1}, \ldots, X_{n-1}$. There is an obvious action of the group algebra $\mathbb{F}\left[F_{n-1}\right]$ on the spectral sequence: simply define the action of $X_{i}$ on $E_{r}$ to be $\alpha_{r}^{\zeta_{i}}$, the map induced by $a^{\zeta_{i}}$ on $E_{r}$ and extend this to $\mathbb{F}\left[F_{n-1}\right]$ in the natural way. Thus an element such as $X_{1} X_{2}+X_{6}$ acts on $E_{r}$ by the chain map $\alpha_{r}^{\zeta_{1}} \circ \alpha_{r}^{\zeta_{2}}+\alpha_{r}^{\zeta_{6}}$. The fact that for each curve $\zeta_{i}$ and each $r$,

$$
\alpha_{r}^{\zeta_{i}}:\left(E_{r}, \delta_{r}\right) \rightarrow\left(E_{r}, \delta_{r}\right),
$$

is a chain map satisfying, $\alpha_{r+1}^{\zeta_{i}}=\left(\alpha_{r}^{\zeta_{i}}\right)_{*}$, implies that this is indeed an action by $\mathbb{F}\left[F_{n-1}\right]$. To see that the action descends to $\Lambda_{n-1}^{*}$, it suffices to check that $X_{i} X_{j}+X_{j} X_{i}$ and $X_{i}^{2}$ act as zero on each page of the spectral sequence, for all $i, j$. These relations clearly hold on the $E_{1}$ page, however, since Theorem 4.5 implies that the map induced by $a^{\zeta_{i}}$ on $E_{1}$ agrees with $x_{i}$, the map on the reduced Khovanov 
complex induced by $p_{i}$. But if an endomorphism of a spectral sequence is zero on some page, it is zero for all subsequent pages, since $\alpha_{r+1}=\left(\alpha_{r}\right)_{*}$. It follows that $X_{i} X_{j}+X_{j} X_{i}$ acts as zero on $E_{r}$ for all $r \geq 1$.

To show that the module structure on $E_{2}$ agrees with the module structure on Khovanov homology induced by the basepoint maps it suffices to note, again, that $\alpha_{1}^{\zeta_{i}}=x_{i}$ and $\alpha_{2}^{\zeta_{i}}=\left(\alpha_{1}^{\zeta_{i}}\right)_{*}=\left(x_{i}\right)_{*}$.

It is natural to ask about convergence of the $\Lambda_{n-1}^{*}$-action. In particular, since the homology of the spectral sequence converges to the Floer homology of $\Sigma(L)$, one could hope that the action converges to the $\Lambda^{*}\left(H_{1}(Y ; \mathbb{F})\right)$-action (where the homology classes $\left[\zeta_{i}\right]$ serve as a spanning set for $\left.H_{1}(Y ; \mathbb{F})\right)$. While this is true it is not necessarily as useful as one may think, since convergence is phrased in terms of the associated graded module (see [20]) and this module may have many extensions. Put differently, the module structure on the associated graded is sensitive only to the lowest order terms of the filtered chain maps $a^{\zeta_{i}}$, and the higher order terms may contribute in a nontrivial way to the module structure on $\widehat{H F}(\Sigma(L))$. Despite this, the corollary can still be used to prove the following "collapse result" for the spectral sequence. This theorem will be one of our main tools for showing that the Khovanov module detects unlinks.

Proposition 4.7 Suppose that $K h^{r}(\bar{L}) \cong \Lambda_{n-1}^{*}$ as a module over $\Lambda_{n-1}^{*}$. Then $\widehat{H F}(\Sigma(L)) \cong \Lambda_{n-1}^{*}$ as a module over $\Lambda^{*}\left(H_{1}(\Sigma(L) ; \mathbb{F})\right)$.

Proof The assumption on the reduced Khovanov homology implies, in particular, that the entire Khovanov homology is supported in homological grading zero. This is because $1 \in K h^{r}(L)$ generates the homology as a module over $\Lambda_{n-1}^{*}$, and the module action preserves the homological grading (it is induced by chain maps of degree zero).

In the proof of Theorem 4.5, however, the filtration on the complex computing $\widehat{H F}(\Sigma(L))$ arises from a graded decomposition coming from the norm of a multiframing

$$
X\left(\{0,1\}^{l}\right)=\bigoplus_{i \geq 0} C^{(i)}, \quad C^{(i)}=\bigoplus_{\left\{I \in\{0,1\}^{l}|| I \mid=i\right\}} \widehat{C F}(Y(I)),
$$

and the norm $|I|$ corresponds to the homological grading on the Khovanov complex. Thus the $E_{2}$ page of the spectral sequence, which is identified with $K h^{r}(L)$, is supported in a single filtration. Since the higher differentials strictly lower the filtration, it follows that the spectral sequence has collapsed and that $K h^{r}(\bar{L}) \cong E_{2}=E_{\infty} \cong \widehat{H F}(\Sigma(L))$. Moreover, since the module structure on $E_{\infty}$ is induced by $E_{2}$ through homology, it follows that this is an isomorphism of $\Lambda_{n-1}^{*}$ modules. We claim that the latter module structure agrees with the $\Lambda^{*}\left(H_{1}(\Sigma(L) ; \mathbb{F})\right)$-module structure on Floer homology. 
To see this, note first that the curves $\zeta_{i}$ span $H_{1}(\Sigma(L) ; \mathbb{F})$. Now on one hand we have the filtered chain maps $a^{\zeta_{i}}$ whose induced maps on homology agree with $A^{\left[\zeta_{i}\right]}: \widehat{H F}(\Sigma(L)) \rightarrow \widehat{H F}(\Sigma(L))$. On the other we have $X_{i}$, the maps induced by $a^{\zeta_{i}}$ on the $E_{\infty}$ page of the spectral sequence. To see that $A^{[\zeta]}=X_{i}$, it suffices to recall the construction from Section 4.1 of the morphism of spectral sequences induced by $a^{\zeta_{i}}$. By construction, each filtered map $a^{\zeta_{i}}$ is chain homotopic to a map

$$
a_{\infty}^{\zeta_{i}}: E_{\infty} \longrightarrow E_{\infty}, \quad a_{\infty}^{\zeta_{i}}=a_{\infty}^{(0)}+a_{\infty}^{(1)}+a_{\infty}^{(2)}+\cdots
$$

and the morphism induced on the $E_{\infty}$ is, by definition, the lowest order term in this map: $\alpha_{\infty}^{\zeta_{i}}:=a_{\infty}^{(0)}$. But the discussion above indicates that $E_{\infty}$ is supported in a single filtration summand, hence the higher order terms in the decomposition of $a_{\infty}^{\zeta_{i}}$ vanish. It follows that the maps $A^{[\zeta]}=\left(a^{\zeta_{i}}\right)_{*}=a_{\infty}^{\zeta_{i}}$ and $X_{i}=a_{\infty}^{(0)}$ are equal. The proposition follows.

\section{A nontriviality theorem for homology actions}

In this section we prove a nontriviality result for Floer homology, Theorem 5.1. Roughly speaking, the theorem says that the homology of the Floer homology with respect to the action of any curve is nontrivial, provided a manifold does not contain a homologically essential 2-sphere. This detection theorem for Floer homology will transfer through the spectral sequence of the previous section to our detection theorem for Khovanov homology.

Following Kronheimer and Mrowka [18], let

$$
\begin{aligned}
& H F^{\circ}(Y \mid R)=\bigoplus H F^{\circ}(Y, \mathfrak{s}), \\
& \left\{\mathfrak{s} \in \operatorname{Spin}^{c}(Y) \mid\left\langle c_{1}(\mathfrak{s}),[R]\right\rangle=x(R)\right\}
\end{aligned}
$$

where $R$ is a Thurston norm minimizing surface in $Y$ and $x(R)$ is its Thurston norm.

Theorem 5.1 Suppose $Y$ is a closed, oriented and irreducible 3-manifold with $b_{1}(Y)>0$. Let $R \subset Y$ be a Thurston norm minimizing connected surface. Then there exists a cohomology class $[\omega] \in H^{2}(Y ; \mathbb{Z})$ with $\langle[\omega],[R]\rangle>0$, such that for any $[\zeta] \subset H_{1}(Y ; \mathbb{Z})$ the homology group with respect to $A^{[\zeta]}$

$$
H\left(\underline{\hat{H F}}\left(Y \mid R ; \mathcal{R}_{[\omega]}\right), A^{[\zeta]}\right)
$$

has positive rank as an $\mathcal{R}$-module. 
Proof We adapt the argument of Ozsváth and Szabó [22, Theorem 4.2].

By Gabai [8], there exists a taut foliation $\mathscr{F}$ of $Y$, such that $R$ is a compact leaf of $\mathscr{F}$. The work of Eliashberg and Thurston [6] shows that $\mathscr{F}_{F}$ can be approximated by a weakly symplectically semifillable contact structure $\xi$, where $Y \times[-1,1]$ is the weak semifilling.

By Giroux [9], $Y$ has an open book decomposition which supports $\xi$. By plumbing positive Hopf bands to the page of such an open book, we may assume that the binding is connected and that the genus of the page is greater than one. Let $K \subset Y$ denote the binding, and let $Y_{0}$ be the fibered 3-manifold obtained from $Y$ by 0 -surgery on $K$. There is a 2-handle cobordism $W_{0}: Y \rightarrow Y_{0}$. Similarly, there is a 2-handle cobordism $-W_{0}: Y_{0} \rightarrow Y$, obtained by reversing the orientation of $W_{0}$ and viewing it "backwards."

Eliashberg [5] shows that the weak semifilling $Y \times[-1,1]$ can be embedded in a closed symplectic 4-manifold $X$ (see also Etnyre [7], for an alternative construction). This is done by constructing symplectic caps for the boundary components. Eliashberg's caps are produced by first equipping the 2-handle cobordisms $W_{0}$ and $-W_{0}$ with appropriate symplectic structures, and then extending these structures over Lefschetz fibrations $V_{0}$ and $V_{0}^{\prime}$ whose boundaries are the fibered 3-manifolds $-Y_{0}$ and $Y_{0}$, respectively (note $V_{0}$ and $V_{0}^{\prime}$ are not, in general, orientation-reversing diffeomorphic). Moreover, [5, Theorem 1.3] says that we can choose $V_{0}$ and $V_{0}^{\prime}$ so that

$$
H_{1}\left(V_{0}\right)=H_{1}\left(V_{0}^{\prime}\right)=0 .
$$

The result of the construction is a closed symplectic 4 -manifold, $(X, \omega)$, which decomposes as

$$
X=V_{0}^{\prime} \cup_{-Y_{0}}-W_{0} \cup_{-Y=Y \times\{-1\}} Y \times[-1,1] \cup_{-Y} W_{0} \cup_{-Y_{0}} V_{0} .
$$

We view the cobordism from right to left, so that the orientation shown on a 3-manifold is that which it inherits as the oriented boundary of the 4-manifold to the right of the union in the decomposition. By perturbing $\omega$ slightly and multiplying by an integer, we may assume $[\omega] \in H^{2}(X ; \mathbb{Z})$. We can also arrange that $b_{2}^{+}\left(V_{0}^{\prime}\right)>1$ and $b_{2}^{+}\left(V_{0}\right)>1$, and hence can decompose $V_{0}$ by an admissible cut along a 3-manifold, $N$. Thus $X=X_{1} \cup_{N} X_{2}$ with $b_{2}^{+}\left(X_{i}\right)>0$, and

$$
X_{2}=V_{0}^{\prime} \cup_{-Y_{0}}-W_{0} \cup_{-Y} Y \times[-1,1] \cup_{-Y} W_{0} \cup_{-Y_{0}}\left(V_{0} \backslash X_{1}\right) .
$$

Denote the canonical $\operatorname{Spin}^{c}$ structure associated to $\omega$ by $\mathfrak{k}(\omega)$, and the restriction of $\mathfrak{k}(\omega)$ to $Y_{0}$ by $\mathfrak{t}$. Let $c(\xi ;[\omega]) \in \underline{\widehat{H F}}\left(-Y \mid R ; \mathcal{R}_{[\omega]}\right) / \mathcal{R}^{\times}$be the twisted Ozsváth and Szabó contact invariant defined in [26;22], and let $c^{+}(\xi ;[\omega]) \in \underline{H F}^{+}\left(-Y \mid R ; \mathcal{R}_{[\omega]}\right) / \mathcal{R}^{\times}$ 
be its image under the natural map $\iota_{*}: \underline{\widehat{H F}} \rightarrow \underline{H F}^{+}$. Let $\pi: Y_{0} \rightarrow S^{1}$ be the fibration on the 0-surgery induced by the open book decomposition of $Y$, and let $c(\pi)$ be a generator of $\underline{\widehat{H F}}\left(-Y_{0}, \mathfrak{t} ; \mathcal{R}_{[\omega]}\right)$ whose image in $\underline{H F}^{+}\left(-Y_{0}, \mathfrak{t} ; \mathcal{R}_{[\omega]}\right) \cong \mathcal{R}$ is a generator $c^{+}(\pi ;[\omega])$. By [26, Proposition 3.1],

$$
\widehat{\widehat{F}}_{W_{0} ;[\omega]}(c(\pi)) \doteq c(\xi ;[\omega]),
$$

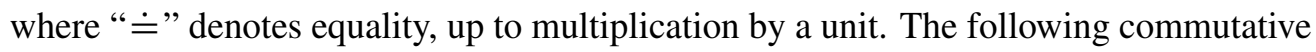
diagram summarizes the relationship between the contact invariants:

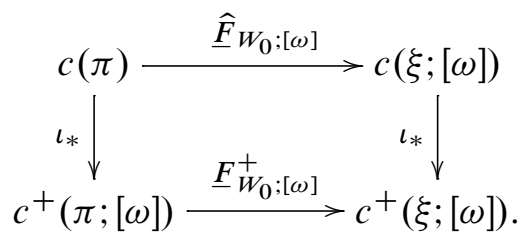

Let $W=V_{0}^{\prime} \cup-W_{0}$, and let $B$ be an open 4-ball in $V_{0}^{\prime}$. As $W$ is a symplectic filling of $(Y, \xi)$, the argument in [22, Proof of Theorem 4.2] shows that

$$
\underline{F}_{W \backslash B ;[\omega]}^{+}\left(c^{+}(\xi ;[\omega])\right)
$$

is a nontorsion element in $\underline{H F}^{+}\left(S^{3} ; \mathcal{R}_{[\omega]}\right)$. Note that here, as above, we regard $W$ from right to left; namely, as a cobordism from $-Y$ to $S^{3}$. It follows that

$$
\widehat{\widehat{F}}_{W \backslash B ;[\omega]}(c(\xi ;[\omega])) \text { is nontorsion. }
$$

One can construct a Heegaard diagram for $-Y_{0}$ such that there are only two intersection points representing $\mathfrak{t}$, and such that there are no holomorphic disks connecting them which avoid the hypersurface specified by the basepoint. Indeed, such a Heegaard diagram is constructed in the course of the proof of [26, Theorem 1.1], and its desired properties are verified in the proof of [26, Proposition 3.1] (here we use the fact that the page of the open book has genus greater than one, though $\mathrm{Wu}[36$, Section 3, specifically Remark 3.3] indicates that the same technique can be adapted for genus one open books). Since $H_{1}(Y ; \mathbb{Z})$ is naturally a subgroup of $H_{1}\left(Y_{0} ; \mathbb{Z}\right)$, any $[\zeta] \in H_{1}(Y)$ can be viewed as an element in $H_{1}\left(Y_{0} ; \mathbb{Z}\right)$, and the preceding discussion implies that

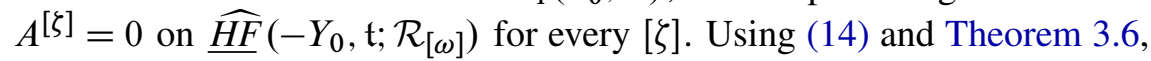

$$
\begin{aligned}
A^{[\zeta]}(c(\xi ;[\omega])) & \doteq A^{[\zeta]} \circ \underline{\hat{F}}_{W_{0} ;[\omega]}(c(\pi)) \\
& =\widehat{\widehat{F}}_{W_{0} ;[\omega]} \circ A^{[\zeta]}(c(\pi)) \\
& =\underline{\widehat{F}}_{W_{0} ;[\omega]}(0)=0 .
\end{aligned}
$$

Hence $c(\xi ;[\omega]) \in \operatorname{ker}\left(A^{[\zeta]}\right)$. 
Now if $k c(\xi ;[\omega]) \in \operatorname{im}\left(A^{[\xi]}\right)$ for some nonzero $k \in \mathcal{R}$, then there is an element $a \in \underline{\widehat{H F}}\left(Y ; \mathcal{R}_{[\omega]}\right)$ such that $A^{[\zeta]}(a)=k c(\xi ;[\omega])$. Using (13) and Theorem 3.6,

$$
\begin{aligned}
\underline{\hat{F}}_{W \backslash B ;[\omega]}(k c(\xi ;[\omega])) & =\underline{\widehat{F}}_{W \backslash B ;[\omega]} \circ A^{[\zeta]}(a) \\
& =A^{0} \circ \underline{\widehat{F}}_{W \backslash B ;[\omega]}(a)=0,
\end{aligned}
$$

a contradiction to (15). Hence $k c(\xi ;[\omega]) \notin \operatorname{im}\left(A^{[\zeta]}\right)$. It follows that $c(\xi ;[\omega])$ represents a nontorsion element in $H\left(\underline{\widehat{H F}}\left(Y \mid R ; \mathcal{R}_{[\omega]}\right), A^{[\xi]}\right)$, so our conclusion holds.

Corollary 5.2 Suppose $Y$ is a closed, oriented 3-manifold which does not contain $S^{1} \times S^{2}$ connected summands. Then there exists a cohomology class $[\omega] \in H^{2}(Y ; \mathbb{Z})$ such that for any $[\zeta] \subset H_{1}(Y ; \mathbb{Z})$ the homology group with respect to $A^{[\zeta]}$

$$
H\left(\underline{\hat{H F}}\left(Y ; \mathcal{R}_{[\omega]}\right), A^{[\zeta]}\right)
$$

has positive rank as an $\mathcal{R}$-module.

Proof In the case that $b_{1}(Y)>0$, this follows from Theorem 5.1 and the twisted version of (5). When $b_{1}(Y)=0$, the theorem holds with $[\omega]=0$. Indeed, when $[\omega]=0$ the corresponding Floer homology group $\widehat{H F}\left(Y ; \mathcal{R}_{[\omega]}\right)$ is simply the homology of $\widehat{C F}(Y) \otimes_{\mathbb{F}} \mathbb{F}\left[T, T^{-1}\right]$; that is, we take the untwisted complex and tensor over $\mathbb{F}$ with $\mathbb{F}\left[T, T^{-1}\right]$. Now [24, Proposition 5.1] indicates that the Euler characteristic of $\widehat{H F}(Y)$, and hence its rank over $\mathbb{F}$, is nontrivial. The universal coefficient theorem then implies $H_{*}\left(\widehat{C F}(Y) \otimes_{\mathbb{F}} \mathbb{F}\left[T, T^{-1}\right]\right)$ has positive rank as an $\mathbb{F}\left[T, T^{-1}\right]$-module. Finally, since every class $[\zeta] \in H_{1}(Y ; \mathbb{Z})$ is torsion, Lemma 3.1 shows that the $A^{[\zeta]}=0$, as an operator on $\widehat{H F}(Y ; \mathcal{R})$.

\section{Links with the Khovanov module of an unlink}

We now bring together the results from previous sections to prove our main theorems. The first task is to prove Theorem 4, which states that Heegaard Floer homology, as a module over $\Lambda^{*}\left(H^{1}(Y ; \mathbb{F})\right)$, detects $S^{1} \times S^{2}$ summands in the prime decomposition of a closed oriented 3-manifold. By way of the module structure on the spectral sequence from Khovanov homology to Heegaard Floer homology (specifically Proposition 4.7), this detection theorem will quickly lead to the Khovanov module's detection of unlinks, Theorem 2.

The detection theorem for the Heegaard Floer module makes use of Corollary 5.2 from the previous section. The main challenge is to take this corollary, which is a nonvanishing result for homology actions on Heegaard Floer homology with twisted 
coefficients, and use it to obtain a characterization result for the Floer homology module with untwisted, ie $\mathbb{F}$, coefficients. Not surprising, to pass from twisted coefficients to untwisted coefficients we will need the universal coefficients theorem. Let us recall its statement from Spanier [34].

Theorem 6.1 Let $C$ be a free chain complex over a principal ideal domain, $R$, and suppose that $M$ is an $R$-module. Then there is a functorial short exact sequence

$$
0 \longrightarrow H_{q}(C) \otimes_{R} M \longrightarrow H_{q}(C ; M) \longrightarrow \operatorname{Tor}_{R}\left(H_{q-1}(C), M\right) \longrightarrow 0 .
$$

This exact sequence is split, but the splitting may not be functorial.

We wish to apply the universal coefficients theorem to understand the Heegaard Floer homology with $\mathbb{F}$ coefficients through an understanding of the Floer homology with twisted coefficients, where the twisted coefficient ring is $\mathcal{R}=\mathbb{F}\left[T, T^{-1}\right]$. Viewing $\mathbb{F}$ as the trivial $\mathcal{R}$ module (where $T$ acts as 1 ), the following lemma analyzes the tensor product in the universal coefficient splitting.

Lemma 6.2 Suppose $M$ is a finitely generated module over $\mathcal{R}=\mathbb{F}\left[T, T^{-1}\right], M^{\text {tors }}$ is the submodule of $M$ consisting of all torsion elements, and $M^{\text {free }}=M / M^{\text {tors }}$. Then there is a short exact sequence

$$
0 \longrightarrow M^{\text {Tors }} \otimes_{\mathcal{R}} \mathbb{F} \longrightarrow M \otimes_{\mathcal{R}} \mathbb{F} \longrightarrow M^{\text {free }} \otimes_{\mathcal{R}} \mathbb{F} \longrightarrow 0 .
$$

Moreover,

$$
M^{\text {tors }} \otimes_{\mathcal{R}} \mathbb{F} \cong \operatorname{Tor}_{\mathcal{R}}(M, \mathbb{F})
$$

Proof The short exact sequence

$$
0 \longrightarrow M^{\text {Tors }} \longrightarrow M \longrightarrow M^{\text {free }} \longrightarrow 0
$$

gives rise to a long exact sequence

$$
\cdots \longrightarrow \operatorname{Tor}_{\mathcal{R}}\left(M^{\text {free }}, \mathbb{F}\right) \longrightarrow M^{\text {Tors }} \otimes_{\mathcal{R}} \mathbb{F} \longrightarrow M \otimes_{\mathcal{R}} \mathbb{F} \longrightarrow M^{\text {free }} \otimes_{\mathcal{R}} \mathbb{F} \longrightarrow 0 .
$$

Since $M^{\text {free }}$ is free, $\operatorname{Tor}_{\mathcal{R}}\left(M^{\text {free }}, \mathbb{F}\right)=0$, hence we have the desired short exact sequence.

Since $\mathcal{R}$ is a principal ideal domain

$$
M^{\mathrm{tors}} \cong \bigoplus_{i} \mathcal{R} /\left(p_{i}^{k_{i}}\right)
$$


where the $p_{i}$ are prime elements in $\mathcal{R}, k_{i} \in \mathbb{Z}_{\geq 1}$. Note that $\mathbb{F} \cong \mathcal{R} /(T-1)$. If $p_{i} \neq(T-1)$ up to a unit, then

$$
\mathcal{R} /\left(p_{i}^{k_{i}}\right) \otimes_{\mathcal{R}} \mathbb{F}=0, \quad \operatorname{Tor}_{\mathcal{R}}\left(\mathcal{R} /\left(p_{i}^{k_{i}}\right), \mathbb{F}\right)=0 .
$$

If $p_{i}=(T-1)$ up to a unit, then

$$
\mathcal{R} /\left(p_{i}^{k_{i}}\right) \otimes_{\mathcal{R}} \mathbb{F} \cong \mathbb{F}, \quad \operatorname{Tor}_{\mathcal{R}}\left(\mathcal{R} /\left(p_{i}^{k_{i}}\right), \mathbb{F}\right) \cong p_{i}^{k_{i}-1} \mathcal{R} /\left(p_{i}^{k_{i}}\right) \cong \mathbb{F} .
$$

Hence our result follows.

Theorem 4 states that if $\widehat{H F}(Y ; \mathbb{F}) \cong \mathbb{F}\left[X_{1}, \ldots, X_{n-1}\right] /\left(X_{1}^{2}, \ldots, X_{n-1}^{2}\right)$ as a module, then $Y \cong M \#\left(\#^{n-1}\left(S^{1} \times S^{2}\right)\right)$, where $M$ is an integer homology sphere satisfying $\widehat{H F}(M) \cong \mathbb{F}$. We turn to the proof of this theorem.

Proof of Theorem 4 We first reduce to the case that $Y$ is irreducible. Suppose that $Y$ is a nontrivial connected sum. Then we can apply (5) to restrict our attention to a connected summand. If $Y=S^{1} \times S^{2}$, then our conclusion holds. So we may assume that $Y$ is irreducible.

Let $\zeta_{1}, \ldots, \zeta_{n-1}$ be elements in $H_{1}(Y ; \mathbb{Z}) /$ Tors such that $A^{\zeta_{i}}(\mathbf{1})=X_{i}$. If we have $S=\left\{i_{1}, \ldots, i_{k}\right\} \subset\{1, \ldots, n\}$, let

$$
A^{S}=A^{\zeta_{i_{1}}} \circ \cdots \circ A^{\zeta_{i_{k}}}
$$

and let

$$
X_{S}=X_{i_{1}} X_{i_{2}} \cdots X_{i_{k}} \in \widehat{H F}(Y ; \mathbb{F}) \cong \Lambda_{n-1}^{*} .
$$

It follows that $A^{S}(\mathbf{1})=X_{S}$. Since $A^{\left[\zeta_{i}\right]}$ decreases the Maslov grading by 1 , we can give a relative Maslov grading to $\widehat{H F}(Y ; \mathbb{F})$ such that the grading of $X_{S}$ is $n-1-|S|$.

By Corollary 5.2, there exists $[\omega] \in H^{2}(Y ; \mathbb{Z})$ such that $H\left(\underline{\widehat{H F}}\left(Y ; \mathcal{R}_{[\omega]}\right), A^{[\zeta]}\right)$ is nontorsion for any $[\zeta] \in H_{1}(Y)$. Let $C=\widehat{\widehat{C F}}\left(Y ; \mathcal{R}_{[\omega]}\right)$. Let $H^{\text {tors }}(C)$ be the submodule of $H(C)$ which consists of all torsion elements in $H(C)$, and let $H^{\text {free }}(C)=$ $H(C) / H^{\text {tors }}(C)$. By Theorem 6.1, there is a short exact sequence

$$
0 \longrightarrow H_{*}(C) \otimes_{\mathcal{R}} \mathbb{F} \stackrel{\mu_{*}}{\longrightarrow} H_{*}\left(C \otimes_{\mathcal{R}} \mathbb{F}\right) \stackrel{\tau_{*}}{\longrightarrow} \operatorname{Tor}_{\mathcal{R}}\left(H_{*-1}(C), \mathbb{F}\right) \longrightarrow 0 .
$$

Moreover, by the functoriality of (16), the three groups are $\Lambda_{n-1}^{*}$-modules such that the exact sequence respects the module structure. Recall that

$$
H_{*}\left(C \otimes_{\mathcal{R}} \mathbb{F}\right) \cong \widehat{H F}(Y ; \mathbb{F}) \cong \Lambda_{n-1}^{*} .
$$

Claim 1 The map $\mu_{n-1}$ is zero. 
If the map $\mu_{n-1}$ is nonzero, then it is an isomorphism since $H_{n-1}\left(C \otimes_{\mathcal{R}} \mathbb{F}\right)$ is onedimensional. Since $1 \in H_{n-1}\left(C \otimes_{\mathcal{R}} \mathbb{F}\right)$ generates the module $H_{*}\left(C \otimes_{\mathcal{R}} \mathbb{F}\right)$, the map $\mu_{*}$ is surjective. Hence $\mu_{*}$ is an isomorphism and $\operatorname{Tor}_{\mathcal{R}}(H(C), \mathbb{F})=0$. Lemma 6.2 implies that $H^{\text {tors }}(C) \otimes_{\mathcal{R}} \mathbb{F}=0$ and the module $H(C) \otimes_{\mathcal{R}} \mathbb{F}$ is isomorphic to the module $H^{\text {free }}(C) \otimes_{\mathcal{R}} \mathbb{F}$. Now we have

$$
H\left(H^{\text {free }}(C) \otimes_{\mathcal{R}} \mathbb{F}, A^{\zeta_{1}}\right) \cong H\left(H(C) \otimes_{\mathcal{R}} \mathbb{F}, A^{\zeta_{1}}\right) \cong H\left(H\left(C \otimes_{\mathcal{R}} \mathbb{F}\right), A^{\zeta_{1}}\right) \cong 0,
$$

a contradiction to the fact that $H\left(H(C), A^{\zeta_{1}}\right)$ has positive rank.

Claim 2 The map $\tau_{1}$ is zero.

If $\tau_{1}$ is nonzero, then there exists an $(n-2)$-element subset $S \subset\{1, \ldots, n-1\}$ such that $\tau_{1}\left(X_{S}\right) \neq 0$. We claim that the $2^{n-2}$ elements

$$
A^{S^{\prime}} \circ \tau(\mathbf{1}), \quad S^{\prime} \subset S,
$$

are linearly independent over $\mathbb{F}$. In fact, suppose $S_{1}, \ldots, S_{m}$ are subsets of $S$ with $\left|S_{i}\right|=k$, we want to show that

$$
\sum_{i} A^{S_{i}} \circ \tau(\mathbf{1}) \neq 0
$$

Apply $A^{S \backslash S_{1}}$ to the left hand side of (17). Since $\left(S \backslash S_{1}\right) \cap S_{i} \neq \varnothing$ for all $i \neq 1$, $A^{S \backslash S_{1}} A^{S_{i}}=0$ when $i \neq 1$. So we get

$$
\begin{aligned}
A^{S \backslash S_{1}}\left(\sum_{i} A^{S_{i}} \circ \tau(\mathbf{1})\right) & =A^{S \backslash S_{1}} A^{S_{1}} \circ \tau(\mathbf{1}) \\
& =A^{S} \circ \tau(\mathbf{1}) \\
& =\tau \circ A^{S}(\mathbf{1}) \\
& =\tau\left(X_{S}\right) \neq 0 .
\end{aligned}
$$

So (17) holds.

Now we have proved that the rank of $\operatorname{Tor}_{\mathcal{R}}(H(C), \mathbb{F})$ is at least $2^{n-2}$, which is half of the rank of $H_{*}\left(C \otimes_{\mathcal{R}} \mathbb{F}\right)$. By (16), we have

$$
H_{*}\left(C \otimes_{\mathcal{R}} \mathbb{F}\right) \cong\left(H(C) \otimes_{\mathcal{R}} \mathbb{F}\right) \oplus \operatorname{Tor}_{\mathcal{R}}(H(C), \mathbb{F}) .
$$

Using Lemma 6.2, we see that $H^{\text {free }}(C) \otimes_{\mathcal{R}} \mathbb{F}=0$, which contradicts the fact that $H(C)$ has positive rank. This finishes the proof of Claim 2.

By Claim 2 we have $\operatorname{Tor}_{\mathcal{R}}\left(H_{0}(C), \mathbb{F}\right)=0$. So

$$
H_{0}^{\text {tors }}(C) \otimes_{\mathcal{R}} \mathbb{F}=0
$$


by Lemma 6.2. By Claim 1,

$$
H_{n-2}^{\text {tors }}(C) \otimes_{\mathcal{R}} \mathbb{F} \cong \operatorname{Tor}_{\mathcal{R}}\left(H_{n-2}(C), \mathbb{F}\right) \neq 0 .
$$

Let $u \in H_{n-2}^{\text {tors }}(C) \otimes_{\mathcal{R}} \mathbb{F}$ be a nonzero element, then $\mu(u) \neq 0$ and there exists an $(n-2)$-element subset $S \subset\{1, \ldots, n-1\}$ such that $A^{S} \circ \mu(u)$ is the generator of $H_{0}\left(C \otimes_{\mathcal{R}} \mathbb{F}\right)$. Thus $\mu \circ A^{S}(u) \neq 0$.

Since $u \in H_{n-2}^{\text {tors }}(C) \otimes_{\mathcal{R}} \mathbb{F}, A^{S}(u) \in H_{0}^{\text {tors }}(C) \otimes_{\mathcal{R}} \mathbb{F} \cong 0$ by (18). Thus $\mu \circ A^{S}(u)=0$, a contradiction.

With the detection theorem in hand, we can easily prove that the Khovanov module detects unlinks.

Proof of Theorem 2 It follows from the module structure of $K h(L)$ that we have $K h^{r}\left(L, L_{0}\right) \cong \Lambda_{n-1}^{*}=\mathbb{F}\left[X_{1}, \ldots, X_{n-1}\right] /\left(X_{1}^{2}, \ldots, X_{n-1}^{2}\right)$. By Proposition 4.7, we have $\widehat{H F}(\Sigma(L)) \cong \Lambda_{n-1}^{*}$ as a module.

By Theorem $4, \Sigma(L) \cong M \#\left(\#^{n-1}\left(S^{1} \times S^{2}\right)\right)$, where $M$ is an integral homology sphere with $\widehat{H F}(M) \cong \mathbb{F}$. If a link $J$ is nonsplit, then $\Sigma(J)$ does not contain an $S^{1} \times S^{2}$ connected summand; on the other hand, if $J=J_{1} \sqcup J_{2}$, then we have $\Sigma(J)=\Sigma\left(J_{1}\right) \# \Sigma\left(J_{2}\right) \#\left(S^{1} \times S^{2}\right)$ [12, Proposition 5.1]. Applying this fact to the link $L$ at hand, it follows that $L=L_{0} \sqcup L_{1} \sqcup \cdots \sqcup L_{n-1}$. Since we have that $K h\left(J_{1} \sqcup J_{2}\right) \cong K h\left(J_{1}\right) \otimes K h\left(J_{2}\right)$, each $L_{i}$ has rank: $K h\left(L_{i}\right)=2$. It follows from [19] that each $L_{i}$ is an unknot, so $L$ is an unlink.

\section{References}

[1] J A Baldwin, On the spectral sequence from Khovanov homology to Heegaard Floer homology, Int. Math. Res. Not. 2011 (2011) 3426-3470 MR2822178

[2] J A Baldwin, A S Levine, A combinatorial spanning tree model for knot Floer homology, Adv. Math. 231 (2012) 1886-1939 MR2964628

[3] J A Baldwin, O Plamenevskaya, Khovanov homology, open books, and tight contact structures, Adv. Math. 224 (2010) 2544-2582 MR2652216

[4] S Eliahou, L H Kauffman, M B Thistlethwaite, Infinite families of links with trivial Jones polynomial, Topology 42 (2003) 155-169 MR1928648

[5] Y M Eliashberg, A few remarks about symplectic filling, Geom. Topol. 8 (2004) 277293 MR2023279

[6] Y M Eliashberg, W P Thurston, Confoliations, University Lecture Series 13, Amer. Math. Soc. (1998) MR1483314 
[7] J B Etnyre, On symplectic fillings, Algebr. Geom. Topol. 4 (2004) 73-80 MR2023278

[8] D Gabai, Foliations and the topology of 3-manifolds, J. Differential Geom. 18 (1983) 445-503 MR723813

[9] E Giroux, Géométrie de contact: de la dimension trois vers les dimensions supérieures, from: "Proceedings of the International Congress of Mathematicians, Vol. II", (T Li, editor), Higher Ed. Press, Beijing (2002) 405-414 MR1957051

[10] J E Grigsby, S M Wehrli, On the colored Jones polynomial, sutured Floer homology, and knot Floer homology, Adv. Math. 223 (2010) 2114-2165 MR2601010

[11] M Hedden, Khovanov homology of the 2-cable detects the unknot, Math. Res. Lett. 16 (2009) 991-994 MR2576686

[12] M Hedden, Y Ni, Manifolds with small Heegaard Floer ranks, Geom. Topol. 14 (2010) 1479-1501 MR2653731

[13] M Hedden, L Watson, Does Khovanov homology detect the unknot?, Amer. J. Math. 132 (2010) 1339-1345 MR2732349

[14] S Jabuka, T E Mark, Product formulae for Ozsváth-Szabó 4-manifold invariants, Geom. Topol. 12 (2008) 1557-1651 MR2421135

[15] V F R Jones, A polynomial invariant for knots via von Neumann algebras, Bull. Amer. Math. Soc. 12 (1985) 103-111 MR766964

[16] M Khovanov, A categorification of the Jones polynomial, Duke Math. J. 101 (2000) 359-426 MR1740682

[17] M Khovanov, Patterns in knot cohomology, I, Experiment. Math. 12 (2003) 365-374 MR2034399

[18] P B Kronheimer, T S Mrowka, Knots, sutures, and excision, J. Differential Geom. 84 (2010) 301-364 MR2652464

[19] P B Kronheimer, T S Mrowka, Khovanov homology is an unknot-detector, Publ. Math. Inst. Hautes Études Sci. (2011) 97-208 MR2805599

[20] J McCleary, User's guide to spectral sequences, Mathematics Lecture Series 12, Publish or Perish, Wilmington, DE (1985) MR820463

[21] Y Ni, Nonseparating spheres and twisted Heegaard Floer homology, Algebr. Geom. Topol. 13 (2013) 1143-1159 MR3044606

[22] P Ozsváth, Z Szabó, Holomorphic disks and genus bounds, Geom. Topol. 8 (2004) 311-334 MR2023281

[23] P Ozsváth, Z Szabó, Holomorphic disks and knot invariants, Adv. Math. 186 (2004) 58-116 MR2065507

[24] P Ozsváth, Z Szabó, Holomorphic disks and three-manifold invariants: Properties and applications, Ann. of Math. 159 (2004) 1159-1245 MR2113020 
[25] P Ozsváth, Z Szabó, Holomorphic disks and topological invariants for closed threemanifolds, Ann. of Math. 159 (2004) 1027-1158 MR2113019

[26] P Ozsváth, Z Szabó, Heegaard Floer homology and contact structures, Duke Math. J. 129 (2005) 39-61 MR2153455

[27] P Ozsváth, Z Szabó, On Heegaard diagrams and holomorphic disks, from: "European Congress of Mathematics”, (A Laptev, editor), Eur. Math. Soc., Zürich (2005) 769-781 MR2185780

[28] P Ozsváth, Z Szabó, On the Heegaard Floer homology of branched double-covers, Adv. Math. 194 (2005) 1-33 MR2141852

[29] P Ozsváth, Z Szabó, Heegaard diagrams and Floer homology, from: "International Congress of Mathematicians, Vol. II”, (M Sanz-Solé, J Soria, J L Varona, J Verdera, editors), Eur. Math. Soc., Zürich (2006) 1083-1099 MR2275636

[30] P Ozsváth, Z Szabó, Holomorphic triangles and invariants for smooth four-manifolds, Adv. Math. 202 (2006) 326-400 MR2222356

[31] P Ozsváth, Z Szabó, An introduction to Heegaard Floer homology, from: "Floer homology, gauge theory, and low-dimensional topology”, (D A Ellwood, P S Ozsváth, A I Stipsicz, Z Szabó, editors), Clay Math. Proc. 5, Amer. Math. Soc. (2006) 3-27 MR2249247

[32] L P Roberts, On knot Floer homology in double branched covers, Geom. Topol. 17 (2013) 413-467 MR3035332

[33] A Shumakovitch, Torsion of the Khovanov homology arXiv:math/0405474

[34] E H Spanier, Algebraic topology, McGraw-Hill, New York (1966) MR0210112

[35] M B Thistlethwaite, Links with trivial Jones polynomial, J. Knot Theory Ramifications 10 (2001) 641-643 MR1831681

[36] Z Wu, Perturbed Floer homology of some fibered three-manifolds, Algebr. Geom. Topol. 9 (2009) 337-350 MR2482081

Department of Mathematics, Michigan State University

A338 WH, East Lansing, MI 48824, USA

Department of Mathematics, California Institute of Technology

1200 E California Blvd, Pasadena, CA 91125, USA

mhedden@math.msu.edu, yini@caltech.edu

http://www . math.msu . edu/ mhedden/Home.html,

http://www.its.caltech.edu/ yini/

Proposed: Ronald Fintushel

Received: 6 May 2013

Seconded: Robion Kirby, Ronald Stern

Accepted: 15 June 2013 